\title{
Hole dynamics in a spin background: A sum-rule-conserving theory with exact limits
}

\author{
W. Metzner \\ Dipartimento di Fisica, Università "La Sapienza," I-00185 Roma, Italy \\ P. Schmit and D. Vollhardt \\ Institut für Theoretische Physik C, Technische Hochschule Aachen, D-5100 Aachen, Federal Republic of Germany
}

(Received 31 July 1991)

\begin{abstract}
A self-consistent theory is formulated for the dynamics of a hole moving in a $d$-dimensional, quantum-mechanical background of spins at arbitrary temperatures. The contribution of loops in the path of a hole, which are always important in dimensions $d>1$, is given particular attention. We first show that the Green function, thermodynamics, and dynamical conductivity can be determined exactly in the limit $d \rightarrow \infty$. On the basis of this solution, we construct an approximation scheme for the dynamics of a hole in dimensions $d<\infty$, where loops are summed self-consistently to all orders. The resulting theory satisfies the spectral and $f$-sum rules and yields the exact solution for the ferromagnetic background in any dimension $d$. Three types of spin backgrounds are explicitly discussed: ferromagnetic, Néel, and random. In the Néel case the retraceable-path approximation by Brinkman and Rice for the Green function is found to be correct up to order $1 / d^{4}$ for large $d$. Detailed calculations of the density of states $D(\omega)$ and the conductivity $\sigma(\omega)$ of the hole are presented for $d=3$ and $\infty$. A characteristic dependence on the particular type of spin background is found, which is especially pronounced in the case of $\sigma(\omega)$.
\end{abstract}

\section{INTRODUCTION}

The discovery of high-temperature superconductivity ${ }^{1}$ has greatly intensified the interest in the properties of strongly correlated tight-binding electrons near the Mott metal-insulator transition. ${ }^{2}$ In the simplest case such a situation is expected to be describable by the Hubbard model $^{3}$ at almost half-filling and large $U$. For low doping (deviation from half-filling), this can then be mapped 4 onto a model of mobile holes in a background of antiferromagnetically coupled spins, the so-called $t$ - $J$ model. ${ }^{5}$

To leading order in the hole density $\delta$ hole-hole correlations can be neglected and it is sufficient to study the motion of a single hole in a spin background-still a genuine many-particle problem. Clearly, this step is valid only as long as the holes keep sufficiently apart (i.e., no hole-hole binding or phase separation). The question under which circumstances this may happen is being studied intensively ${ }^{6-8}$ at present, but shall not be addressed here. It should be borne in mind that the very derivation of the $t-J$ model from a strongly interacting fermionic lattice model is valid only to leading order in $\delta$. Calculations of hole-hole correlations within the $t$ - $J$ model should therefore be accompanied by an assessment of the influence of corrections of order $\delta^{2}$ to the model itself.

The investigation of the motion of a single hole in a spin system was pioneered by Brinkman and Rice. ${ }^{9}$ They considered several configurations of the spin background (ferromagnetically ordered, Néel ordered, random) and studied the corresponding density of hole states and dc conductivity. Their calculation was based on Nagaoka's ${ }^{10}$ expansion of expectation values in terms of background-conserving hole paths, i.e., paths where the initial and final spin configurations are the same. Summing up the subclass of all paths with no closed loops ("retraceable paths"), Brinkman and Rice 9 obtained an approximation which turned out to be particularly accurate for the single-particle Green function of a hole (e.g., the density of states) in a Néel-ordered background and is exact in $d=1$. Here a "loop" refers to an excursion of the hole away from the retraceable path into the background. Later, the retraceable path (or Brinkman-Rice) approximation was used to calculate many other quant1ties; i.e., Rice and Zhang ${ }^{11}$ evaluated the dynamical conductivity, while Oguri and Maekawa ${ }^{12}$ studied the electrical resistivity, thermal conductivity, thermopower, and specific heat.

Recently, several authors ${ }^{13-15}$ improved the theory of the hole dynamics by taking into account spin fluctuations, which were neglected in the work of Brinkman and Rice. ${ }^{9}$ These are particularly important in lowdimensional $(d=2)$ systems, e.g., those relevant for high$T_{c}$ superconductivity. Simultaneously, there has been a number of numerical studies of the problem in $d=1,2 \mathrm{di}$ mensions, ${ }^{16-19}$ providing an independent check for the theoretical ideas.

In the present work we do not treat the quantum spin fluctuations, but rather concentrate on another correction to the Brinkman-Rice approximation, which is important in any dimension $d>1$ : the contribution of loops. The retraceable-path approximation does not distinguish between different spin backgrounds since paths without loops naturally conserve any initial spin configuration and therefore always contribute. However, while in an antiferromagnetic environment backgroundconserving paths with loops are rare (and thus not overly important), they do become important whenever there 
are clusters of aligned spins. In particular, in a ferromagnetically ordered spin background, any hole path leaves the background unchanged and it is this maximum freedom in the motion which leads to Nagaoka's theorem, ${ }^{10}$ stating that the kinetic energy of a hole becomes minimal in a ferromagnetic configuration. By contrast, if paths with loops are neglected, the kinetic energy is the same for any spin configuration. Some authors ${ }^{12,20}$ have incorporated four-step loops (plaquettes) to improve the Brinkman-Rice approximation and thus managed to discuss the Hall effect and magnetoresistance - two effects where the configuration dependence is crucial. However, to describe correctly the ferromagnetic limit, it is clearly necessary to include loops of arbitrary length. While the ferromagnetic background itself is actually easy to handle, it is a nontrivial task to construct a self-consistent approximation scheme which treats general spin configurations in a unified scheme and which, at the same time, fulfills exact sum rules and also treats the ferromagnetic case correctly.

The approximation we present in this paper is constructed by taking the limit of high dimensions $d \rightarrow \infty$ (Ref. 21) as a guide, since in this case the hole dynamics can be determined exactly. For the Néel-ordered background, one can then show that the single-particle Green function obtained by the Brinkman-Rice approximation ${ }^{9}$ is correct up to order $1 / d^{4}$. Furthermore, for a hole in a general $d$-dimensional spin background, we construct an approximation scheme for the Green function and the dynamical conductivity which has the following properties.

(i) It becomes exact for any spin background in $d=\infty$.

(ii) It yields the exact result for the ferromagnetic background in any dimension.

(iii) The Green function has the correct analytic properties; i.e., the spectral function is never negative and the spectral sum rule is always satisfied.

(iv) The conductivity has the correct analytic properties; i.e., its real part is never negative, and the $f$-sum rule is satisfied.

The paper is structured as follows. In Sec. II we present a precise statement of the problem; in particular, we specify several types of spin backgrounds relevant for a $t-J$ model. Section III is devoted to the calculation of the Green function of a hole in various spin configurations. After reviewing Nagaoka's path formalism and the retraceable-path approximation, we identify the paths contributing in $d=\infty$. We then calculate the exact Green function for a hole in a Néel and random background (including the ferromagnetic configuration as a limiting case) and discuss the properties of the corresponding spectral functions and self-energies. These results are used to construct a conserving approximation for finite dimensions $d$ and are then applied to the case $d=3$. In Sec. IV the thermodynamics of the hole problem is derived from the Green function and the lowtemperature behavior of the specific heat is investigated. The dynamical conductivity is calculated and discussed in Sec. V, following the logical lines of Sec. III. A critical summary including an outline of possible extensions of this work closes the presentation.

\section{DILUTE HOLES IN A $t$ - $J$ MODEL}

For strong repulsion $(U>t)$ and low doping $(\delta<<1)$, the Hubbard model $^{3}$ may be transformed ${ }^{4}$ into the socalled $t-J$ model,

$$
\begin{aligned}
& H_{t J}=H_{t}+H_{J} \\
& H_{t}=-t \sum_{\langle\mathrm{ij}\rangle} \sum_{\sigma}\left(1-n_{\mathrm{i},-\sigma}\right) c_{\mathrm{i}, \sigma}^{\dagger} c_{\mathrm{j}, \sigma}\left(1-n_{\mathrm{j},-\sigma}\right), \\
& H_{J}=J \sum_{\langle\mathrm{ij}\rangle} \mathbf{S}_{\mathrm{i}} \cdot \mathbf{S}_{\mathrm{j}},
\end{aligned}
$$

which is expected to describe the low-temperature properties of strongly interacting tight-binding electrons near a Mott metal-insulator transition. ${ }^{2,5}$ Here $c_{\mathrm{i}, \sigma}^{\dagger}\left(c_{\mathrm{i}, \sigma}\right)$ creates (annihilates) electrons with spin $\sigma$ on a lattice site $\mathrm{i}, n_{\mathrm{i}, \sigma}=c_{\mathrm{i}, \sigma}^{\dagger} c_{\mathrm{i}, \sigma}$ is the corresponding number operator, and $\mathbf{S}_{\mathrm{i}}$ is the spin operator on site $\mathbf{i}$. The underlying Hilbert space is restricted to non-doubly-occupied sites. The kinetic term $H_{t}$ describes restricted hopping of electrons to next-neighbor sites (amplitude $-t$ ), while $H_{J}$ describes an antiferromagnetic $(J>0)$ Heisenberg exchange interaction between next-neighbor spins.

To leading order in an expansion in powers of the hole density $\delta$, the correlations between holes play no role. In this case it is sufficient to study the motion of a single hole in a background of spins. The structure of the spin background strongly depends on the $t-J$ model parameters, lattice structure, and temperature. We consider only hypercubic lattices in $d$ dimensions. Following Brinkman and Rice, ${ }^{9}$ we will discuss the following three types of spin backgrounds.

(i) Ferromagnetic background $(F)$ : As pointed out by Nagaoka, ${ }^{10}$ the $F$ background minimizes the kinetic energy $H_{t}$ of a single hole (as compared to other backgrounds); it is, however, destabilized by $H_{J}$, hole-hole correlations, and the spin entropy (at $T>0$ ). Hence, if at all, ${ }^{22-24}$ the ferromagnetic background is relevant only for $J \ll \delta t$ at low temperatures, where $\delta$ must also be small enough to render the hole-hole correlations unimportant.

(ii) Néel background $(N)$ : For $T, \delta t<<$ and $\delta<<1$, the spins order antiferromagnetically, at least in $d \geq 3$ (in lower dimensions there are at least strong antiferromagnetic correlations in this case). In high dimensions the spin fluctuations become weak (they disappear in $d=\infty$ ) and the antiferromagnetically ordered state may be approximated by a Néel state. ${ }^{25}$ In addition, we will restrict our discussion to the case $J \ll t$, thus neglecting the excitation energy of the spins which are overturned by the moving hole. ${ }^{6,14}$

(iii) Random background $(R)$ : If $T \gg \delta t, J$, all spin configurations appear with equal probability in a thermodynamic ensemble (note that for a single hole $\delta \rightarrow 0$ in the thermodynamic limit and hence $T \gg \delta$ for any $T>0$ ). We will treat a generalization of this case, where the average density of spins $\sigma, p_{\sigma}$, may be different from $p_{-\sigma}$, as would be the case in the presence of a homogenous magnetic field. The ferromagnetic background (i) 
is thereby included as a special case, i.e., $\left|p_{\sigma}-p_{-\sigma}\right| \rightarrow 1$.

For each of the background types $(F, N, R)$, we will calculate and discuss the Green function for a hole inserted into the undoped spin system (Sec. III), the thermodynamics of one-hole states (Sec. IV), and finally, the dynamical conductivity of a hole moving in this background (Sec. V).

\section{GREEN FUNCTION AND DENSITY OF STATES}

In this section we calculate the Green function for a hole inserted into a spin background of types $F, N$, and $R$, respectively:

$$
\begin{aligned}
& G_{\mathrm{ij}}^{X}(z)=\sum_{\sigma} G_{\sigma \mathrm{ij}}^{X}(z), \\
& G_{\sigma \mathrm{ij}}^{X}(z)=\left\langle c_{\mathrm{i}, \sigma}^{\dagger} \frac{1}{z-H} c_{\mathrm{j}, \sigma}\right\rangle_{X} .
\end{aligned}
$$

Here $H=H_{t}$ [Eq. (1b)] and $\langle\cdots\rangle_{X}$, with $X \in\{F, N, R\}$, denotes the background average, i.e.,

$$
\begin{aligned}
& \langle\cdots\rangle_{F}=\langle F|\cdots| F\rangle, \\
& |F\rangle=\prod_{\mathrm{i}} c_{\mathrm{i}, \sigma}^{\dagger}|0\rangle,
\end{aligned}
$$

for the ferromagnetic background ( $|0\rangle$ is the vacuum),

$$
\begin{aligned}
& \langle\cdots\rangle_{N}=\langle N|\cdots| N\rangle, \\
& |N\rangle=\left(\prod_{i \in A} c_{i \uparrow}^{\dagger}\right)\left(\prod_{i \in B} c_{i \downarrow}^{\dagger}\right)|0\rangle,
\end{aligned}
$$

for the Néel background ( $A$ and $B$ are the two sublattices of the hypercubic lattice), and

$$
\langle\cdots\rangle_{R}=\sum_{s} w_{s}\langle s|\cdots| s\rangle
$$

for the random background (we set $\hbar=1$ ); $w_{s}$ is a normalized $\left(\Sigma_{s} w_{s}=1\right)$ distribution of spin configurations $s$ such that spins on different sites are statistically independent and the probability (or average density) for spins $\sigma$ is $p_{\sigma}$. Note that in the unpolarized case $\left(p_{\uparrow}=p_{\downarrow}=\frac{1}{2}\right),\langle\cdots\rangle_{R}$ is just the thermal average

$$
\langle\cdots\rangle_{R}=\sum_{s}\left\langle s\left|e^{-\beta H} \cdots\right| s\right\rangle / \sum_{s}\left\langle s\left|e^{-\beta H}\right| s\right\rangle,
$$

since $H|s\rangle=0$ in our case (all spin configurations therefore contribute with equal weight $w_{s}$ ). For $p_{\sigma} \neq p_{-\sigma}$, however, $\langle\cdots\rangle_{R}$ corresponds to a thermal average in the presence of a magnetic field. The Green function determines the density of states $D^{X}(\omega)$ via

$$
D^{X}(\omega)=\frac{1}{\pi} \operatorname{Im} G^{X}\left(\omega-i 0^{+}\right),
$$

where $G^{X}(z) \equiv G_{\mathrm{ii}}^{X}(z)$ is the local Green function (which is independent of $\mathrm{i}$ in all cases $F, N$, and $R$ ). For these three backgrounds the hole Green function is translationally invariant; the Fourier transform of $G_{\mathrm{ij}}^{X}(z)$ will be denoted by $G^{X}(\mathbf{k}, z)$, and we define the spectral function $\rho^{X}(\mathbf{k}, \omega)$ via

$$
\rho^{X}(\mathbf{k}, \omega)=\frac{1}{\pi} \operatorname{Im} G^{X}\left(\mathbf{k}, \omega-i 0^{+}\right)
$$

and the self-energy $\Sigma_{\sigma}^{X}(\mathbf{k}, z)$ by

$$
G^{X}(\mathbf{k}, z)=\sum_{\sigma} \frac{1}{z+\varepsilon_{\mathbf{k}}-\Sigma_{\sigma}^{X}(\mathbf{k}, z)},
$$

where $\varepsilon_{\mathbf{k}}=-2 t\left(\cos k_{1}+\cdots+\cos k_{d}\right)$ is the dispersion relation corresponding to $H_{t}$ on a hypercubic lattice, with the lattice constant set equal to unity; the choice of the plus sign for $\varepsilon_{\mathbf{k}}$ in (9) will become clear below.

\section{A. Nagaoka paths and retraceable-path approximation}

Using the identity

$$
\frac{1}{z-H}=\frac{1}{z} \sum_{n=0}^{\infty}(H / z)^{n}
$$

Nagaoka ${ }^{10}$ derived an expansion of the local Green function $G_{\mathrm{ii}}^{s}(z)$ for a hole inserted into an arbitrary, fixed spin configuration $|s\rangle$,

$$
G_{\mathrm{ii}}^{s}(z) \equiv \sum_{\sigma}\left\langle s\left|c_{\mathrm{i}, \sigma}^{\dagger} \frac{1}{z-H} c_{\mathrm{i}, \sigma}\right| s\right\rangle,
$$

in powers of $t / z$. He found

$$
G_{\mathrm{ii}}^{s}(z)=\frac{1}{z}+\frac{1}{z} \sum_{n=2}^{\infty} A_{\mathrm{i} n}^{s}(-t / z)^{n},
$$

where $A_{\mathrm{i} n}^{s}$ is the number of distinct $n$-step paths of a hole in the spin background $s$ which start and end on site $\mathbf{i}$ and which restore the original spin configuration after the last step. Note that the final configuration is required to be equal to the original one only up to a permutation of equal spins. However, since paths contributing to $A_{\text {in }}^{s}$ on a hypercubic lattice involve only permutations with even parity, all paths sum up with positive sign. Minus signs may appear for other lattices (think, for example, of a hole moving around a triangle on a triangular lattice).

A straightforward generalization of (12) to the nonlocal part of the Green function $G_{\mathrm{ij}}^{s}(z)$ reads

$$
G_{\mathrm{ij}}^{s}(z)=\frac{1}{z} \delta_{\mathrm{ij}}+\frac{1}{z} \sum_{n=1}^{\infty} A_{\mathrm{ijn}}^{s}(-t / z)^{n},
$$

where $\boldsymbol{A}_{\mathrm{ij} n}^{s}$ is the number of $n$-step paths from $\mathbf{j}$ to $\mathbf{i}$ which leave $s$ invariant; here paths with odd $n$ (contributing if $\mathbf{i}$ and $\mathbf{j}$ are separated by an odd number of nextneighbor steps on the lattice) involve odd permutations and therefore contribute negatively.

The Green function for a hole in a ferromagnetic background $G_{\mathrm{ij}}^{F}(z)$ is now easily evaluated. In a ferromagnetic background any hole path leaves the spin configuration invariant; i.e., the hole behaves like a free particle on an empty lattice, except for the fact that paths with an odd number of steps have a negative phase factor. Looking at (13), one realizes that this extra minus sign corresponds to simply letting $t \rightarrow-t$. Since the free-particle Green function is

$$
G^{0}(\mathbf{k}, z)=\frac{1}{z-\varepsilon_{\mathbf{k}}},
$$

one finds, for the ferromagnetic case, 


$$
G^{F}(\mathbf{k}, z)=\frac{1}{z+\varepsilon_{\mathbf{k}}} .
$$

In real space this means

$$
G_{\mathrm{ij}}^{F}(z)=(-1)^{\mathrm{i}-\mathrm{j}} G_{\mathrm{ij}}^{0}(z),
$$

where $(-1)^{\mathbf{i}-\mathbf{j}}$ is $+1(-1)$ if $\mathbf{i}$ and $\mathbf{j}$ are separated by an even (odd) number of lattice steps. In particular, the density of states of the hole corresponds to that for a free particle:

$$
D^{F}(\omega)=D^{0}(\omega) .
$$

For a general spin background (in particular, for the $N$ and $R$ backgrounds), the contribution of paths can no longer be calculated exactly to all orders $n$. In this situation Brinkman and Rice ${ }^{9}$ evaluated the local Green function by summing all paths without loops (retraceablepath approximation). Naturally, these special paths are background restoring for any spin configuration; i.e., the results of this approximation do not depend on the spin background at all. Comparison with the results of a moment expansion of the density of states ${ }^{9}$ shows that the retraceable-path approximation is particularly accurate for the single-particle Green function of a hole moving in the Néel background. In fact, in this case the contributions of loops are strongly suppressed by the requirement of background conservation. The leading correction enters at order $(t / z)^{12}$ since on the smallest loop (a plaquette) a hole must circulate 3 times to restore the original Néel spin configuration. ${ }^{9}$ The retraceable-path approximation is exact a Bethe lattice, where loops are $a$ priori absent.

For backgrounds other than Néel, on a hypercubic lattice in $d>1$, the retraceable-path approximation is less satisfactory. For example, it does not take into account the fact that ferromagnetic clusters favor hole motion (thus leading to Nagaoka's theorem ${ }^{10}$ ) and cannot describe the Hall effect and magnetioresistance, since it does not distinguish between different spin configurations. Loops become important whenever the spins on a loop on the lattice may be aligned (as in the case of $F$ and $R$ backgrounds); in this case it is sufficient for the hole to circulate through the loop once to restore the spin configuration on the loop.

Our aim in the following is to construct a selfconsistent approximation for the Green function where loops are included to all orders and the ferromagnetic case is treated exactly.

\section{B. Limit of high lattice dimensions}

The limit of high lattice dimensions $d \rightarrow \infty$, introduced only recently to models of correlated fermions, ${ }^{21}$ has helped to clarify the validity of several well-known approximations and to construct new ones. ${ }^{26}$ As will be shown below, the dynamics of a hole in a $F, N$, or $R$ background can be calculated exactly in $d=\infty$ and the resulting theory can be directly extended to finite dimensions, where it provides an approximation with several attractive features (correct sum rules, correct analytic behavior, correct ferromagnetic limit). To keep the average

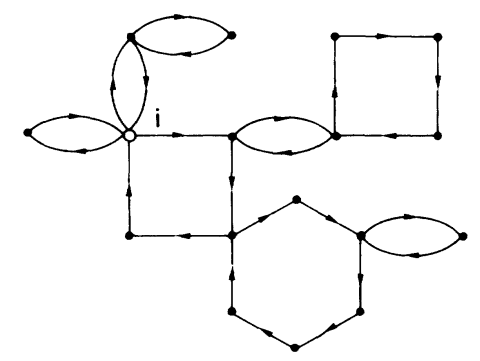

FIG. 1. Typical path of a hole contributing to $G_{\mathrm{ii}}^{s}(z)$ in $d=\infty$; the hole starts and ends on site i. Lines connect next neighbors on a $d$-dimensional lattice.

kinetic energy finite in $d \rightarrow \infty$, one must scale the hopping amplitude $t$ as ${ }^{21}$

$$
t=\frac{t^{*}}{\sqrt{Z}}, t^{*} \text { fixed, }
$$

where $Z$ is the number of nearest neighbors $(Z=2 d$ on a hypercubic lattice). The density of states of a free particle moving on an empty lattice in $d \rightarrow \infty$ becomes a Gaussian distribution with finite width:

$$
D^{0}(\omega)=\frac{1}{t^{*} \sqrt{2 \pi}} e^{-\omega^{2} / 2 t^{* 2}} .
$$

The scaling (18) will be employed throughout this paper. We start by considering the paths contributing to the local Green function $G_{\mathrm{ii}}^{s}(z)$ for an arbitrary spin configuration $s$. In $d=\infty$ the only relevant paths are fully two-particle-reducible clusters of loops ("loop trees"), as indicated in Fig. 1, in which a hole moves along a loop only once. By counting the number of possible embeddings of various path topologies on the lattice, it is easy to see that paths with loops on which a hole walks around more than once or with multiply connected loops (as, for example, in Fig. 2) are suppressed by some integer power of $1 / d$ with respect to the loop tree (for a more detailed discussion, see Ref. 27).

The question of whether or not a hole path restores the spin configuration is easily settled for loop trees. The spin configuration is restored if and only if all spins in a given loop are equal except for the site where the hole enters the loop, in which case the spin direction is unimportant. An example for such a spin configuration is shown in Fig. 3.

The paths contributing to the nonlocal part of the Green function $G_{\mathrm{ij}}^{s}(z)$ in $d=\infty$ are chains (from $\mathrm{j}$ to $\mathrm{i}$ ) decorated by loop trees, as shown in Fig. 4. Oneparticle-irreducible insertions as those in Fig. 5 are suppressed by some integer power of $1 / d$. Therefore,

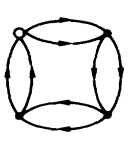

(a)

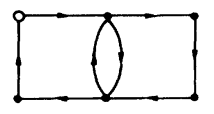

(b)
FIG. 2. (a) Loop on which a hole must walk around more than once; (b) multiply connected loop. Both paths contribute to order $1 / d^{2}$. 


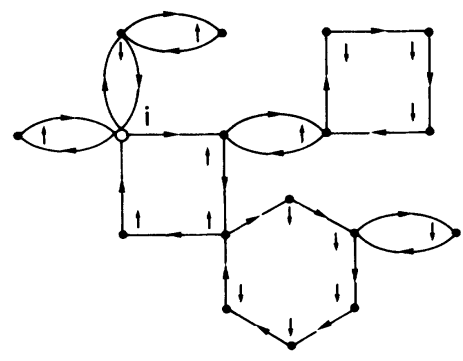

FIG. 3. Spin configuration for which the indicated path is background restoring.

when the hole has traveled from $j$ to $i$, the spin configuration is restored if and only if all spins on the chain are aligned (apart from the above condition for the loops, of course).

At this stage it is already quite clear that the hole dynamics can be calculated exactly in $d=\infty$. We now turn to the explicit summation of all paths contributing in the case of the Néel and random background in this limit.

\section{Néel background}

As discussed above, in $d=\infty$ only loop trees with loops on which a hole circulates around once contribute to the local Green function for a hole. On the other hand, a hole moving around a loop only once destroys the Néel configuration. Hence, for a Néel background in $d=\infty$, loops do not contribute at all and the retraceablepath approximation is exact in this case. Furthermore, the nonlocal part of the Green function is zero for a Néel background in $d=\infty$ since a Néel configuration contains no chains of aligned spins. Taking the limit $d \rightarrow \infty$ in the result of the retraceable-path summation ${ }^{9}$ for the local (i.e., diagonal) Green function, one obtains

$$
G_{\mathrm{ij}}^{N}(z)=\frac{z}{2 t^{* 2}}\left[1-\left(1-4 t^{* 2} / z^{2}\right)^{1 / 2}\right] \delta_{\mathrm{ij}} .
$$

This is the exact Green function for a single hole moving in a Néel spin background in $d=\infty$. The corresponding density of states is

$$
D^{N}(\omega)=\frac{1}{2 \pi t^{* 2}}\left(4 t^{* 2}-\omega^{2}\right)^{1 / 2}, \quad|\omega| \leq 2 t^{*} .
$$

Note that $D^{N}$ has a semielliptic shape, i.e., has sharp

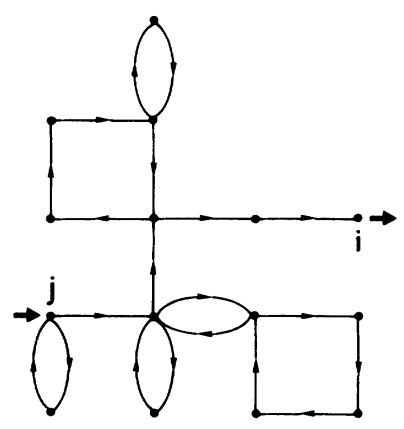

FIG. 4. Typical hole path contributing to $G_{\mathrm{ij}}^{s}(z)$ in $d=\infty$; the hole enters at site $\mathbf{j}$ and leaves at site $\mathbf{i}$.

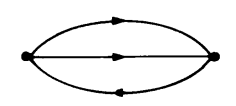

FIG. 5. One-particle-irreducible insertion, yielding an extra factor $1 / d$ as compared to a single line.

band edges (at $\pm 2 t^{*}$ ) with a square-root singularity. This is quite different from the density of states of a hole in a ferromagnetic background in $d=\infty$ [Eq. (17)], which has exponential tails extending to $\omega \rightarrow \pm \infty$. It should be noted that (21) corresponds to the density of states of a hole on a Bethe lattice with an infinite number of nearest neighbors.

The spectral function $\rho^{N}(\mathbf{k}, \omega)$ is $\mathbf{k}$ independent here, since $G_{\mathrm{ij}}^{N}(z)$ is diagonal. In particular, there is no quasiparticle peak. Clearly, the Green function $G_{\mathrm{ij}}^{N}(z)[\mathrm{Eq}$. (20)] is analytic in the upper and lower complex $z$ plane, $D^{N}(\omega)$ being positive and its integral being 1 , as it should. ${ }^{28}$

We close this subsection by pointing out that for the Néel state the corrections to the retraceable-path approximation enter only at order $1 / d^{4}$ in a $1 / d$ expansion. These corrections are due to plaquettes which, as already mentioned in Sec. III A, a hole must walk around 3 times to restore the Néel configuration. Hence there are $3 \times 4=12$ hopping steps required, each of them carrying an amplitude $t=t^{*} /(2 d)^{1 / 2}$, while the number of embeddings of the plaquette on a $d$-dimensional lattice is proportional to $d(d-1)$. This makes the contribution of the plaquette of order $d^{2} / d^{12 / 2}=1 / d^{4}$.

\section{Random background}

In the random case every path contributes to the Green function; i.e., for each hole path there are (many) spin configurations which are restored after the last step. We now sum up all these contributions exactly in $d=\infty$.

We first calculate the local Green function $G^{R}(z)$ in the random ensemble [Eq. (5)] for general $p_{\uparrow}, p_{\downarrow}$. In $d=\infty$ it is sufficient to sum up all the loop trees. To this end we group paths with the same topology into classes which we call graphs. The contribution of a path to $G^{R}$ is given by the probability for restoring the (random) spin configuration, which, for loop trees, is just the probability for finding the spins within each loop to be aligned (except for the site where the hole enters the loop; see Sec. III B). This probability, $p_{g}$, is clearly the same for different paths with the same graph. To obtain the contribution of a graph $g$, it is therefore sufficient to multiply $p_{g}$ by the number of embeddings $m_{g}$ of the graph on the lattice (where different vertices of the graph have to occupy different lattice sites). For loop trees in $d \rightarrow \infty, m_{g}$ is just the product of the number of embeddings of each loop on the tree. The factor $(-t / z)^{n}$ in (12) is treated by associating a factor $-t / z$ with each line of a graph. Thus the local random Green function becomes

$$
G^{R}(z)=\frac{1}{z}+\frac{1}{z} \sum_{g} v_{g}(z),
$$

where the sum extends over all loop tree graphs $g$, and $v_{g}$, 


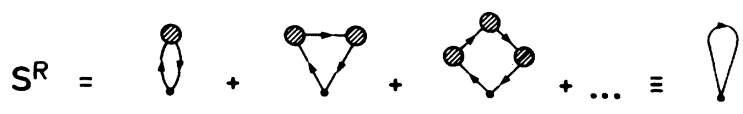

FIG. 6. $S^{R}$ in terms of dressed vertices $C^{R}$.

the "value" of a graph $g$, is given by

$$
v_{g}=m_{g} p_{g}(-t / z)^{n},
$$

with $n$ as the number of lines in $g$. To sum up all graphs, we introduce a quantity $S^{R}$, defined by

$$
G^{R}(z)=\frac{1}{z\left[1-S^{R}(z)\right]} .
$$

Expanding this in a geometric series in $S^{R}$, a comparison with (22) shows that $S^{R}$ is given by the sum over all graphs for which the hole returns to the starting point only once. ${ }^{29}$

Because of the simple topology of the loop trees, $S^{R}$ can be written as a sum over loops with dressed vertices $C^{R}$, as indicated in Fig. 6. The dressed vertex $C^{R}$ is given by the bare vertex plus all possible $S^{R}$ insertions, as shown in Fig. 7. Using the rules for the calculation of the graph values $v_{g}$, one obtains, from Figs. 6 and 7, respectively,

$$
\begin{aligned}
& S^{R}(z)=\sum_{\sigma} \sum_{n=2}^{\infty} u_{n}\left[C_{\sigma}^{R}(z)\right]^{n-1}(t / z)^{n}, \\
& C_{\sigma}^{R}(z)=p_{\sigma} \sum_{l=0}^{\infty}\left[S^{R}(z)\right]^{l}=\frac{p_{\sigma}}{1-S^{R}(z)} .
\end{aligned}
$$

Here $u_{n}$ is the number of self-avoiding return paths on the lattice. For a hypercubic lattice in $d$ dimensions, $u_{2}=2 d, u_{3}=0, u_{4}=2 d(2 d-2)$, etc. Note that the neglect of loops, i.e., setting $u_{n}=0$ for $n \geq 3$ in (25), is equivalent to the retraceable-path approximation and hence leads to the result $G^{R}=G^{N}$, i.e., to the Néel state, with $G^{N}$ given by (20). Defining a generating function for the numbers $u_{n}$ via

$$
M(\xi)=1+\sum_{n=2}^{\infty} u_{n} \xi^{n}
$$

Eq. (25) can be written as

$$
S^{R}(z)=\sum_{\sigma} \frac{M\left(C_{\sigma}^{R}(z) t / z\right)-1}{C_{\sigma}^{R}(z)} .
$$

Eliminating $C_{\sigma}^{R}$ and $S^{R}$ from (24), (26), and (28), one obtains a single equation for $G^{R}$ :

$$
\sum_{\sigma} p_{-\sigma} M\left(p_{\sigma} t G^{R}\right)=1+p_{\uparrow} p_{\downarrow}\left(z G^{R}-1\right) .
$$

Clearly, $M(\xi)$ is not an elementary function. However,

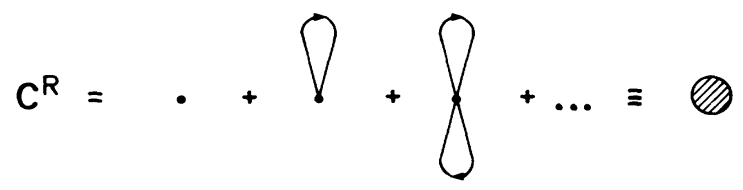

FIG. 7. $C^{R}$ in terms of $S^{R}$. we can relate it to the (already known) function

$$
G^{F}(z)=G^{0}(z)=\int d \varepsilon \frac{D^{0}(\varepsilon)}{z-\varepsilon},
$$

by taking the limit $p_{\uparrow} \rightarrow 1$ (i.e., $p_{\downarrow} \rightarrow 0$ ) in (29); using $M(\xi)=1+O\left(\xi^{2}\right)$ for small $\xi$, this yields $M\left(t G^{0}\right)={ }_{z} G^{0}$ and, consequently,

$$
M(\xi)=\frac{\xi}{t} G_{-1}^{0}(\xi / t),
$$

where $G_{-1}^{0} \equiv\left(G^{0}\right)^{-1}$ is the inverse function of $G^{0}[\mathrm{Eq}$ (30)]. Inserting $M$ [Eq. (31)] into (29), we obtain our final result for $G^{R}(z)$ :

$$
p_{\uparrow} p_{\downarrow}\left[z G^{R}-1-G^{R} \sum_{\sigma} G_{-1}^{0}\left(p_{\sigma} G^{R}\right)\right]+1=0 .
$$

In particular, for the unpolarized random background $\left(p_{\uparrow}=p_{\downarrow}=\frac{1}{2}\right)$, this can be simplified to

$$
G^{R}=2 G^{0}\left(\frac{z}{2}+\frac{3}{2 G^{R}}\right) .
$$

Note that (32) determines the exact local Green function of a hole in a random spin background in $d \rightarrow \infty$.

As to the nonlocal part of $G_{\mathrm{ij}}^{R}(z)$, we must sum all selfavoiding chains extending from $\mathbf{j}$ to $\mathbf{i}$, which may be dressed by arbitrary loop trees (see Sec. III B). The local decorations can again be collected into a dressed vertex $C_{\sigma}^{R}(z)$. In this way $G_{\mathrm{ij}}^{R}(z)$ can be written as

$$
G_{\mathrm{ij}}^{R}(z)=\frac{1}{z} \delta_{\mathrm{ij}}+\frac{1}{z} \sum_{\sigma} \sum_{q=1}^{\infty} u_{\mathrm{ij}}^{q}\left[C_{\sigma}^{R}(z)\right]^{q+1}(t / z)^{q},
$$

where $u_{\mathrm{ij}}^{q}$ is the number of self-avoiding paths connecting $\mathrm{j}$ to $\mathrm{i}$ by $q$ steps on the lattice. The minus sign appearing in front of $t$ in (13) has been compensated by the sign associated with the permutations of fermions. Inserting $C_{\sigma}^{R}(z)=p_{\sigma} z G^{R}(z)$ and using $G_{\mathrm{ij}}^{0}(z)$ to construct a generating function for the $u_{\mathrm{ij}}^{q}$, one obtains, after Fourier transformation,

$$
\begin{aligned}
G^{R}(\mathbf{k}, z) & =\sum_{\sigma} G_{\sigma}^{R}(\mathbf{k}, z) \\
G_{\sigma}^{R}(\mathbf{k}, z) & =\frac{1}{G_{-1}^{0}\left(p_{\sigma} G^{R}(z)\right)+\varepsilon_{\mathrm{k}}} .
\end{aligned}
$$

Comparison with (9) yields the self-energy, which turns out to be independent of $\mathbf{k}$ :

$$
\Sigma_{\sigma}^{R}=-z-G_{-1}^{0}\left(p_{\sigma} G^{R}(z)\right) .
$$

For $p_{\uparrow}=p_{\downarrow}=\frac{1}{2}$ one may use (33) to simplify (36) to

$$
\Sigma_{\sigma}^{R}(z)=\frac{z}{2}-\frac{3}{2 G^{R}(z)} .
$$

Properties of the solution $G^{R}$ in $d=\infty$ : The exact hole Green function $G^{R}$ in $d=\infty$ is obtained by inserting the free local propagator $G^{0}$ for an infinite-dimensional lattice [given by (19) and (30)] into the equations for $G^{R}$. The resulting density of states $D^{R}(\omega)$ for $p_{\uparrow}=p_{\downarrow}=\frac{1}{2}$ is shown in Fig. 8, where $D^{F}=D^{0}$ and $D^{N}$ are also included 


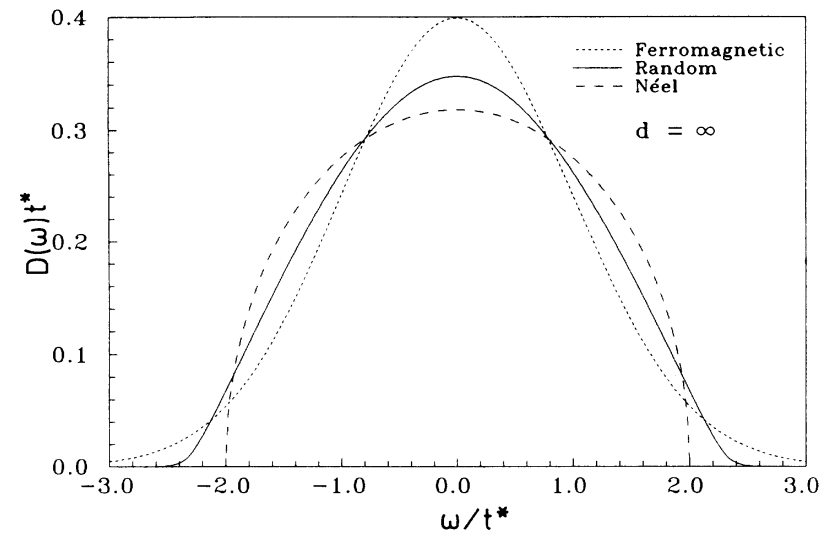

FIG. 8. Exact density of states $D^{R}(\omega)$ for a hole in a random background $\left(p_{\uparrow}=p_{\downarrow}=\frac{1}{2}\right)$ in $d=\infty$. Also shown are the exact results for $D^{F}(\omega)\left[=D^{0}(\omega)\right]$ and $D^{N}(\omega)$ for a ferromagnetic and Néel background, respectively.

for comparison. Here and in all following figures, the energy scale is $t^{*}=\sqrt{2 d} t$. The center of $D^{R}$ is obviously broadened relative to $D^{0} ; D^{R}$ has exponential tails extending to $\pm \infty$, which, however, fall off more rapidly than those of $D^{0}$, i.e.,

$$
D^{R}(\omega) \propto e^{-2 \omega^{2} / t^{* 2}},|\omega| \gg t^{*} .
$$

The states in the tails (for $\omega \rightarrow-\infty$, say) correspond to low-energy hole states due to large ferromagnetic clusters. In a random configuration the probability for the formation of such clusters decays exponentially with its size. Consequently, $D^{R}(\omega)$ is suppressed exponentially with respect to $D^{0}(\omega)$ for $\omega \rightarrow \pm \infty$.

In Fig. 9, $\Sigma^{R}\left(\omega-i 0^{+}\right)$is shown (again $p_{\uparrow}=p_{\downarrow}=\frac{1}{2}$ ). Note, for comparison, that in a ferromagnetic background (all spins up, say) $\Sigma_{\uparrow}^{F}=0$, while $\Sigma_{\downarrow}^{F}=\infty$, which expresses the simple fact that there exist no down spins and therefore one cannot create a hole by removing one. The imaginary part of $\Sigma$ is a measure for the damping of "quasiparticle" peaks in $\rho(\mathbf{k}, \omega)$. Since $\operatorname{Im} \Sigma^{R}(\omega$ $\left.-i 0^{+}\right)>0$ for all $\omega$, there are no genuine quasiparticles in the system. However, for large $|\omega|, \rho^{R}(\mathbf{k}, \omega)$ has sharp peaks of exponentially small width [of order

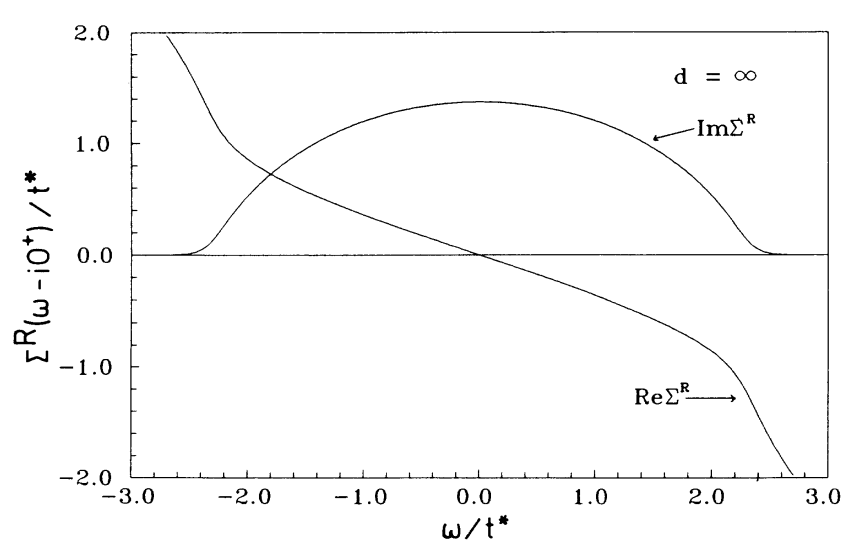

FIG. 9. Real and imaginary parts of the exact self-energy $\Sigma^{R}\left(\omega-i 0^{+}\right)$for the random background $\left(p_{\uparrow}=p_{\downarrow}=\frac{1}{2}\right)$ in $d=\infty$. $\left.\exp \left(-2 \omega^{2} / t^{* 2}\right)\right]$, but their weight decays with the same exponential factor. The asymptotic behavior of the Green function for large $|\omega|$ is (for $p_{\uparrow}=p_{\downarrow}=\frac{1}{2}$ )

$$
G^{R}\left(\omega-i 0^{+}\right) \sim \frac{1}{\omega+\varepsilon_{\mathrm{k}} / 2-i 0^{+}},|\omega| \gg t^{*},
$$

which has the form of the ferromagnetic Green function $G^{F}$, with the hopping amplitude being renormalized by a factor $\frac{1}{2}$. (This is the probability for finding the spin on the site onto which the hole is hopping to be aligned with the previous one.)

\section{E. Self-consistent approximation for finite dimensions}

In finite dimensions $d$ the simplifications arising in the limit $d=\infty$ no longer apply, and the one-hole problem becomes much more complicated; an exact solution for $d>1$ does not exist so far. However, on the basis of our exact results for $d=\infty$, we may construct a selfconsistent approximation for the motion of a hole in a finite-dimensional spin background, in which loops are included up to infinite order and sum rules are fulfilled exactly. This approximation should only be used in $d \geq 3$; in lower dimensions it can no longer be expected to be reliable-besides the fact that spin fluctuations then become crucial.

\section{Néel background}

Here two different approximations are possible, both of which become exact in $d=\infty$. First of all, one may use the results of the retraceable-path approximation, ${ }^{9}$ which not only yields the exact result for $d=1$ and is correct up to order $1 / d^{4}$ for large dimensions in this case, but also fulfills the spectral sum rule in any dimension. In particular, for $d=3$ on a simple cubic lattice one finds ${ }^{9}$

$D^{\mathrm{rpa}}(\omega)=\frac{3}{\pi} \frac{\left(\frac{10}{3} t^{* 2}-\omega^{2}\right)^{1 / 2}}{6 t^{* 2}-\omega^{2}},|\omega| \leq\left(\frac{10}{3}\right)^{1 / 2} t^{*}$

The fact that (40) is correct up to order $1 / d^{4}$ does not imply that for $d=3$, where $1 / d^{4}$ is small, $D^{\text {rpa }}$ is quantitatively close to the exact result for all $\omega$. Indeed, at the band edges, where the density of states is small, corrections become numerically arbitrarily large. Hence all low-temperature properties of the hole depend sensitively on the specific band-edge behavior (see Sec. V E).

An alternative approximation for finite dimensions may be obtained from the formalism developed in Secs. III C and III D. According to the discussion below (26), diagrams with self-avoiding loops do not contribute beyond second order for a Néel background in $d=\infty$. Hence $(20)$ is the exact Green function in this case. In fact, since the entire formalism leading to (20) is selfconsistent and exact in $d=\infty$, we may employ the halfellipse (21) as an approximation even for finite $d$, knowing that, by construction, all sum rules (e.g., the spectral sum rule for the Green function) are fulfilled exactly. The $d$ dependence then only enters through the scaled hopping amplitude $t^{*} / \sqrt{2 d}$. The approximation thus obtained 
will still be denoted by a superscript $N$ ( $\equiv$ Néel). Although this approximation treats the sum rules exactly, the Green function $G_{\mathrm{ij}}(z)$ itself is only correct up to order $t^{4}$, i.e., up to $d\left(1 / d^{4 / 2}\right)=1 / d$ for large $d$, as can be seen from the paths contributing to this approximation. Hence it cannot be expected to be as good an approximation for the density of states (DOS) as the retraceablepath approximation in this particular case.

\section{Random background}

To obtain an approximation for $d<\infty$, one may insert the free, $d$-dimensional local propagator $G^{0}$ into the equations for $G^{R}$ derived in Sec. III D, where loops are included to all orders. Again, the hopping is scaled according to (18). For $\left|p_{\uparrow}-p_{\downarrow}\right| \rightarrow 1, G^{R}$ converges to $G^{F}$; i.e., the ferromagnetic limit is described exactly for all $d$. The approximate Green function $G^{R}(\mathbf{k}, z)$ is analytic in the upper and lower complex $z$ plane, and the spectral density $\rho^{R}(\mathbf{k}, \omega)$ is never negative and satisfies the exact rule $^{28}$

$$
\int \frac{d^{d} k}{(2 \pi)^{d}} \int d \omega \rho(\mathbf{k}, \omega)=1 .
$$

The proof is given in the Appendix.

In Fig. 10 we show the approximate density of states $D^{R}(\omega)$ for a three-dimensional cubic lattice $\left(p_{\uparrow}=p_{\downarrow}=\frac{1}{2}\right)$, in comparison with the respective results for the Néel $\left(D^{N}\right)$ and ferromagnetic $\left(D^{F}=D^{0}\right)$ background, as well as $D^{\text {rpa }}$ obtained from (40). Obviously, $D^{R}$ has the same type of band edge as $D^{0}$ (square-root singularity). The bandwidth of $D^{R}$ is narrower than the free bandwidth. Actually, the exact $D^{R}$ is expected to have exponential tails ${ }^{9}$ extending to the bandwidth of $D^{0}$, these tails being due to the existence of large ferromagnetic clusters in the random ensemble (similar to Lifshitz tails ${ }^{30}$ in the density of states of disordered systems, which are caused by states in large ordered domains). Our approximation does not describe this detail. The approximate selfenergy $\Sigma^{R}\left(\omega-i 0^{+}\right)$in $d=3$ for $p_{\uparrow}=p_{\downarrow}=\frac{1}{2}$ is shown in Fig. 11. Its imaginary part is small only near, or beyond,

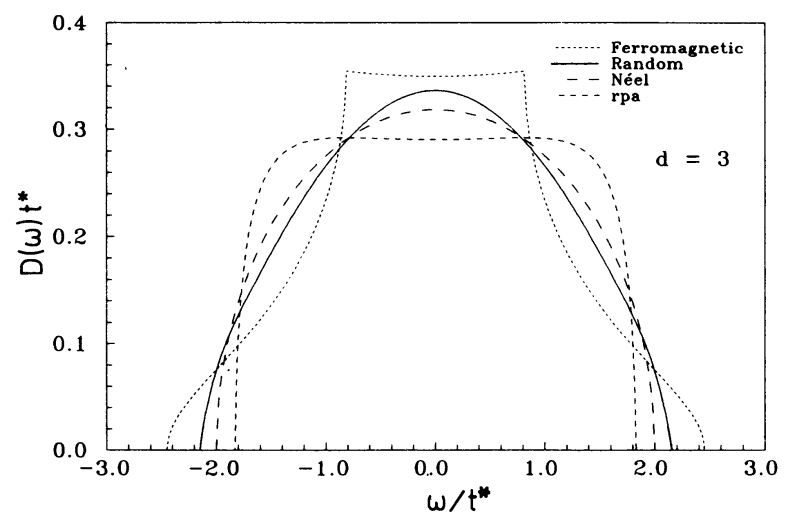

FIG. 10. Density of states for an unpolarized random background (solid line) in $d=3$ as obtained by the self-consistent approximation. The results for the Neel and ferromagnetic (= free) cases, as well as that by the retraceable-path approximation (rpa) are also shown.

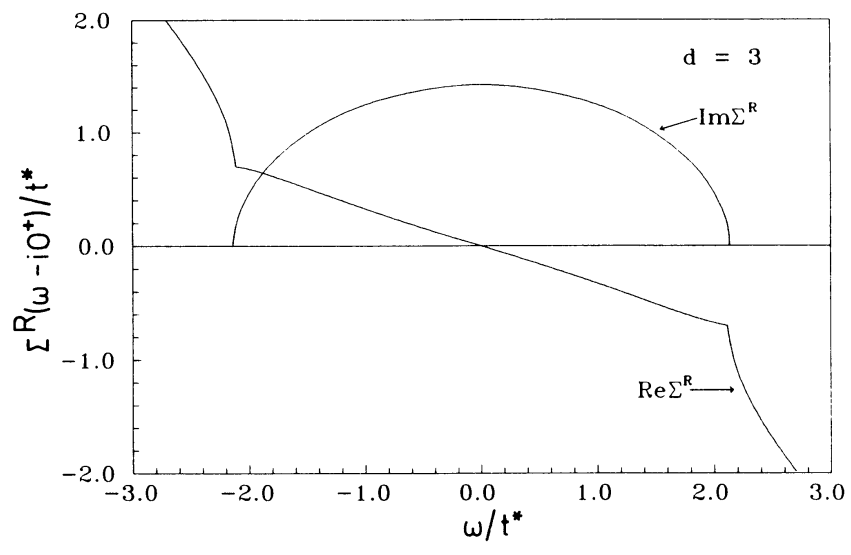

FIG. 11. Real and imaginary parts of the self-consistently determined self-energy $\Sigma^{R}\left(\omega-i 0^{+}\right)$for a random background $\left(p_{\uparrow}=p_{\downarrow}=\frac{1}{2}\right)$ in $d=3$.

the band edge of $D^{R}$, where $D^{R}$ is small, too [this feature follows directly from (37)]. Hence there are no quasiparticle peaks in the spectral function $\rho^{R}(\mathbf{k}, \omega)$ as shown in Fig. 12. This is similar to the behavior found in Ref. 4.

\section{THERMODYNAMICS}

The thermodynamics of single-hole states can be directly calculated from the density of states $D^{X}(\omega)$. The partition function $Z^{X}$ of a hole in a spin background $X$ is given by ${ }^{9,12}$

$$
Z^{X}=L \int d \omega D^{X}(\omega) e^{-\beta \omega},
$$

where $L$ is the number of sites, $\beta=1 / k_{B} T$, is the inverse temperature, and $D^{X}$ is the density of one-hole states in $X$. Note that for $X=F, N$ the assumption of a pure spin configuration is justified only at temperatures which are low with respect to some energy (coupling or external field) stabilizing the magnetic order.

From $Z^{X}$ one obtains the free energy, entropy, internal energy, and specific heat. The free energy

$$
F^{X}=-k_{B} T \ln Z^{X}
$$

and the entropy $S^{X}=-\partial F^{X} / \partial T$ for a single hole diverge

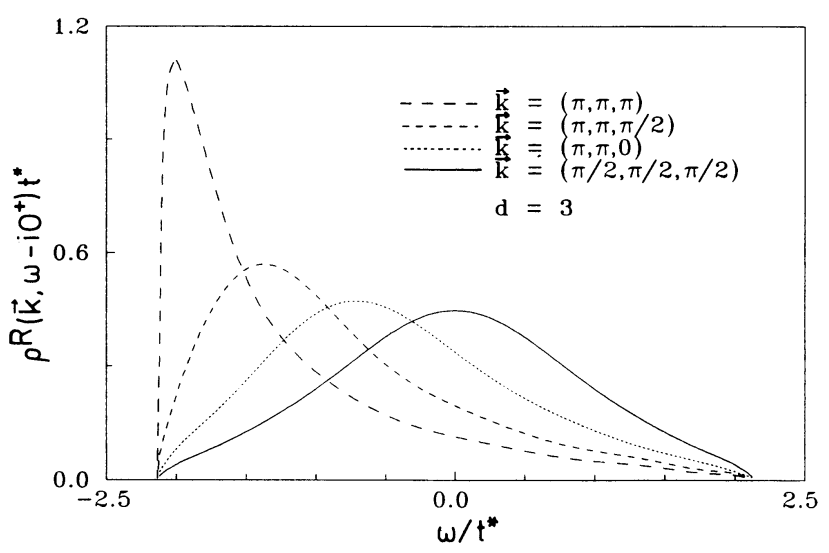

FIG. 12. Spectral function for a hole in an unpolarized random background, $\rho^{R}(\mathbf{k}, \omega)$, in $d=3$ for various $\mathbf{k}$ vectors. 


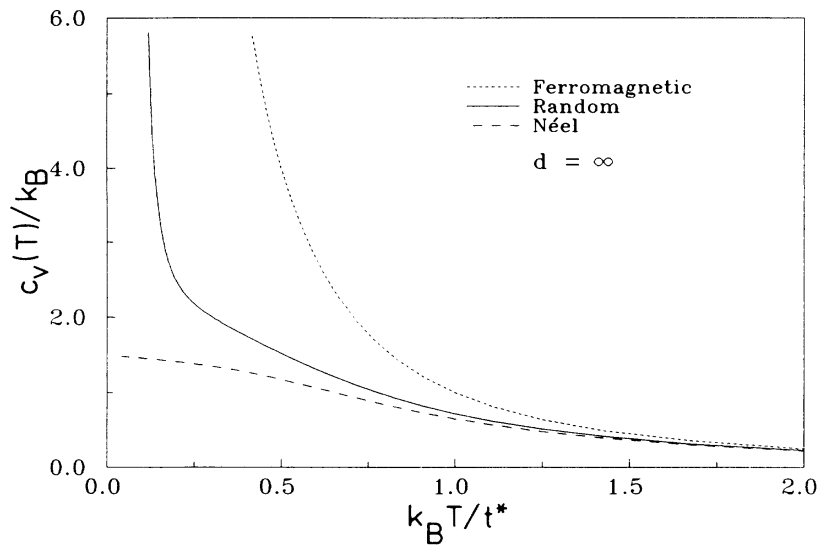

FIG. 13. Exact specific heat of single-hole states vs temperature in $d=\infty$ for various spin backgrounds: ferromagnetic $\left(c_{V}^{F}\right)$, Néel order $\left(c_{V}^{N}\right)$, and random $\left(c_{V}^{R}\right)$.

logarithmically as $L \rightarrow \infty$, while the internal energy $U^{X}=F^{X}+T S^{X}$ and the specific heat $c_{V}^{X}=\partial U^{X} / \partial T$ remain finite. The divergence of $S^{X}$ and $F^{X}$ is due to the divergence of the number of available one-hole states for $L \rightarrow \infty$. To obtain a well-defined thermodynamic limit, one therefore has to introduce a finite hole density $\delta$. Assuming that holes are well separated (i.e., excluding holehole binding, phase separation, etc.) and neglecting holehole correlations (which is correct to leading order in $\delta$ ), one can calculate the free energy and entropy per hole if $L$ is substituted by $\delta^{-1}$ in the expressions for a single hole; here $\delta^{-1}$ is just the average number of sites available for each hole in an infinite system with hole density $\delta$. The internal energy and specific heat per hole is still given by the single-hole expansion (where $L$ does not enter). In $d=\infty$ the internal energies $U^{F}$ and $U^{R}$ go to $-\infty$ as $T \rightarrow 0$, since the spectra of single-hole states are not bounded from below in these cases, but have exponential tails. By contrast, for a Néel background, $U^{N}$ goes to $-2 t^{*}$, the lower band edge of $D^{N}$, at low temperatures. Similarly, the specific heats $c_{V}^{F}$ and $c_{V}^{R}$ diverge for $T \rightarrow 0$, while $c_{V}^{N}$ converges to the finite value $\frac{3}{2} k_{B}$. In Fig. 13 we show $c_{V}^{F}, c_{V}^{R}$ (for $p_{\uparrow}=p_{\downarrow}=\frac{1}{2}$ ), and $c_{V}^{N}$ as functions of $T$ in $d=\infty$.

In $d=3$ our approximation described in Sec. III E always yields densities of states with a square-root-shaped band edge. Consequently, the internal energy $U^{X}$ approaches the lower band edge for $T \rightarrow 0$ in all three cases $X=F, N, R$, while the specific heat always assumes the limiting value $\frac{3}{2} k_{B}$. In Fig. 14 we show $c_{V}^{R}$ as obtained by our approximation (with $p_{\uparrow}=p_{\downarrow}=\frac{1}{2}$ ) in comparison with the exact result for $c_{V}^{F}$ and the approximation for the

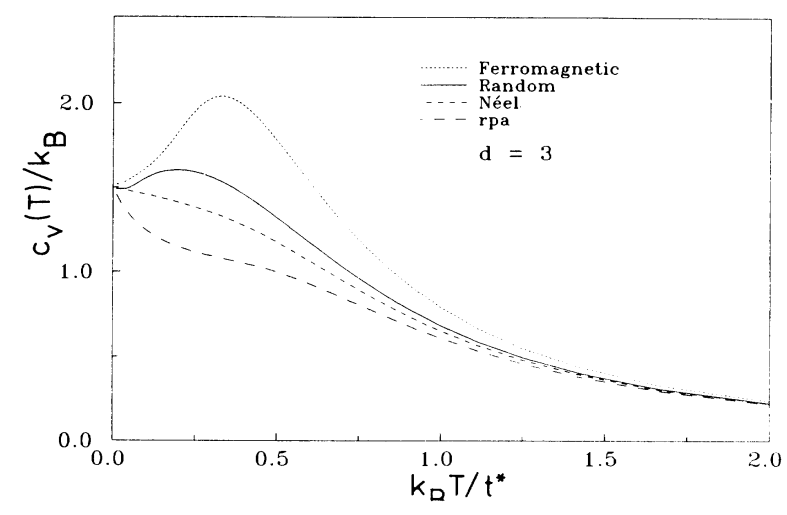

FIG. 14. Specific heat of single-hole states in a random background, $c_{V}^{R}(T)$, for $d=3$ as obtained by the self-consistent approximation. The results of the retraceable-path approximation $c_{V}^{\text {rpa }}$, of the approximation for the Néel state $c_{V}^{N}$, and the exact result for the ferromagnetic background $c_{V}^{F}$ are also shown.

Néel state $c_{V}^{N}$, as well as $c_{V}^{\text {rpa }}$ for a three-dimensional cubic lattice.

\section{DYNAMICAL CONDUCTIVITY}

\section{A. Expansion as a sum over paths}

The dynamical conductivity of a single hole in a spin background $X$ is given by the Kubo formula as

$$
\sigma^{X}(\omega)=V^{-1} \int_{0}^{\infty} d \tau \int_{0}^{\beta} d \lambda\left\langle J_{\alpha}(0) J_{\alpha}(\tau+i \lambda)\right\rangle_{X h} e^{i \omega \tau},
$$

where $V$ is the volume. Here $J_{\alpha}(s)$ is the current operator for a lattice model with next-neighbor hopping amplitude $-t$, i.e.,

$$
\begin{aligned}
& J_{\alpha}(s)=e^{i H s} J_{\alpha} e^{-i H s}, \\
& J_{\alpha}=-i e a t \sum_{\mathrm{j}, \sigma}\left(c_{\mathrm{j}+\alpha, \sigma}^{\dagger} c_{\mathrm{j} \sigma}-c_{\mathrm{j}-\alpha, \sigma}^{\dagger} c_{\mathrm{j} \sigma}\right),
\end{aligned}
$$

where the lattice constant $a$ is now written out explicitly, and $\alpha$ is an arbitrary unit vector on the lattice ( $\sigma^{X}$ does not depend on the direction of $\alpha$ ). Furthermore, $\langle\cdots\rangle_{X h}$ is a thermal average over all single hole states in the background $X$, i.e.,

$$
\langle\cdots\rangle_{X h}=\frac{1}{Z^{X}} \operatorname{tr}_{X h}\left(e^{-\beta H} \cdots\right),
$$

where the trace runs over a complete set of one-hole states in $X$, including an average over all spin configurations in the random case.

Inserting a complete set of one-hole eigenstates of $H$ between the current operators in (44), one can carry out the $\tau$ and $\lambda$ integrals. This yields ${ }^{9,12}$

$$
\sigma^{X}(\omega)=\frac{i}{V Z^{X}} \int \frac{d \omega_{1}}{2 \pi} \int \frac{d \omega_{2}}{2 \pi} \frac{1}{\omega-\omega_{1}+\omega_{2}+i 0^{+}} \frac{e^{-\beta \omega_{1}}-e^{-\beta \omega_{2}}}{\omega_{1}-\omega_{2}} F^{X}\left(\omega_{1}, \omega_{2}\right),
$$

where

$$
F^{X}\left(\omega_{1}, \omega_{2}\right)=\sum_{s_{1}, s_{2}= \pm 1} s_{1} s_{2} f^{X}\left(\omega_{1}+i s_{1} 0^{+}, \omega_{2}+i s_{2} 0^{+}\right)
$$


and the function $f^{X}\left(z_{1}, z_{2}\right)$ is defined by

$$
f^{X}\left(z_{1}, z_{2}\right)=\operatorname{tr}_{X h}\left(\frac{1}{z_{1}-H} J_{\alpha} \frac{1}{z_{2}-H} J_{\alpha}\right) \text {. }
$$

So far, no approximation has been made.

In particular, the real part of the complex conductivity $\sigma(\omega)$ [Eq. (48)] is given by ${ }^{12}$

$$
\operatorname{Re} \sigma^{X}(\omega)=-\frac{1}{4 \pi V Z^{X}} \frac{1-e^{-\beta \omega}}{\omega} \int_{-\infty}^{\infty} d \omega_{1} e^{-\beta \omega_{1}} F^{X}\left(\omega_{1}, \omega_{1}+\omega\right)
$$

Inserting $J_{\alpha}$ [Eq. (46)], one obtains ${ }^{9}$

$$
f^{X}\left(z_{1}, z_{2}\right)=f_{1}^{X}\left(z_{1}, z_{2}\right)+f_{2}^{X}\left(z_{1}, z_{2}\right)
$$

where

$$
\begin{aligned}
& f_{1}^{X}\left(z_{1}, z_{2}\right)=2 e^{2} a^{2} t^{2} \sum_{\langle\mathrm{ij}\rangle} \sum_{\sigma, \sigma^{\prime}} \operatorname{tr}_{X h}\left(\frac{1}{z_{1}-H} c_{\mathrm{i}-\alpha, \sigma}^{\dagger} c_{\mathrm{i} \sigma} \frac{1}{z_{2}-H} c_{\mathrm{j}+\alpha, \sigma^{\prime}}^{\dagger} c_{\mathrm{j} \sigma^{\prime}}\right), \\
& f_{2}^{X}\left(z_{1}, z_{2}\right)=-2 e^{2} a^{2} t^{2} \sum_{\langle\mathrm{ij}\rangle} \sum_{\sigma, \sigma^{\prime}} \operatorname{tr}_{X h}\left(\frac{1}{z_{1}-H} c_{\mathrm{i}+\alpha, \sigma}^{\dagger} c_{\mathrm{i} \sigma} \frac{1}{z_{2}-H} c_{\mathrm{j}+\alpha, \sigma^{\prime}}^{\dagger} c_{\mathrm{j} \sigma^{\prime}}\right) .
\end{aligned}
$$

Using the identity (10) one can expand $f^{X}\left(z_{1}, z_{2}\right)$ as a sum over closed paths, ${ }^{9}$ similar to the expansion of $G^{X}$ in Sec. III A. In Fig. 15 we show the general structure of the hole paths contributing to $f_{1}^{X}$ and $f_{2}^{X}$, respectively. A solid line represents a step $\mathbf{i} \rightarrow \mathbf{i}-\boldsymbol{\alpha}$, etc., in (53) and (54) generated by in the current operator, while a wiggly line represents a sequence of steps generated by $\left(z_{1}-H\right)^{-1}$ or $\left(z_{2}-H\right)^{-1}$. Hence the hole starts at site $\mathrm{i}+\alpha$ and then moves through the segments $1,2,3,4$ of the path, thus returning to $\mathbf{j}+\boldsymbol{\alpha}$. The spins on the sites $\mathbf{j}, \mathbf{i}-\boldsymbol{\alpha}, \mathbf{i}$ in Fig. 15(a) and on $j, i+\alpha, i$ in Fig. 15(b), respectively, must be equal to make possible the restoration of the spin configuration by the moving hole. Note that in general the paths in Fig. 15 may interfere; e.g., the paths labeled by 2 and 4 may have common lattice sites; furthermore, $\mathrm{i}-\boldsymbol{\alpha}$ and $\mathbf{j}$ may be equal, etc. In finite dimension an exact summation of all paths is possible only for the ferromagnetic background.

\section{B. Limit $d \rightarrow \infty$}

In the limit of high lattice dimensions $d \rightarrow \infty$, there are two simplifications in the calculation of $f^{X}$.

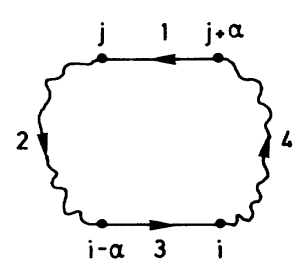

(a)

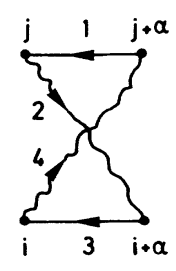

(b)
FIG. 15. General structure of the hole paths contributing (a) to $f_{1}^{X}$ and (b) to $f_{2}^{X}$. The solid lines represent single steps as produced by the current operators; the wiggly lines indicate paths produced by the resolvents $\left(z_{i}-H\right)^{-1}$. The numbers $1,2,3,4$ indicate the sequence of the motion. (i) Interferences between the paths 2 and 4 in Fig. 15 can be neglected.

(ii) $f_{2}^{X}=0$.

This may be understood from the three graphs contributing to $f^{X}$ shown in Fig. 16. Graph (a) contributes to $f_{1}^{X}$ and has no interferences; graph (b) also contributes to $f_{1}^{X}$, but has an interference on site $\mathbf{h}$; graph (c) contributes to $f_{2}^{X}$. All these graphs have the same number of lines; however, while the number of embeddings of graph (a) is of order $d^{3}$ in high dimensions, for (b) and (c) it is only of order $d^{2}$ (steps from $\mathbf{j}+\boldsymbol{\alpha}$ to $\mathbf{j}$, from $\mathbf{i}-\boldsymbol{\alpha}$ to $\mathbf{i}$ and from $\mathrm{i}+\boldsymbol{\alpha}$ to $\mathrm{j}$ have a fixed direction). Hence the contribution of the graphs (b) and (c) is suppressed by a factor $1 / d$ with respect to (a); for $d \rightarrow \infty$ this leads to the two simplifications indicated above.

The absence of interferences between the paths labeled 2 and 4 in Fig. 15 allows one to sum these segments of the total path separately. Clearly, these separate sums are precisely the hole Green function $G^{X}$, e.g., $G_{\mathrm{i}-\boldsymbol{\alpha}, \mathrm{j}}^{X}\left(z_{2}\right)$ in the case of segment 2, in Fig. 15(a). It is therefore possible to evaluate $f^{X}$ exactly in $d=\infty$. In finite dimensions $d$ this is no longer possible. However, the neglect of interferences may be used as an approximation in this case. At the same time the contributions to $f_{2}^{X}$ have to be included to treat the ferromagnetic limit correctly.

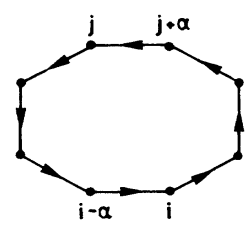

(a)

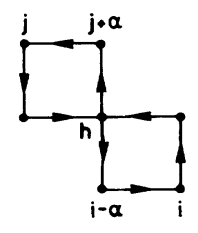

(b)

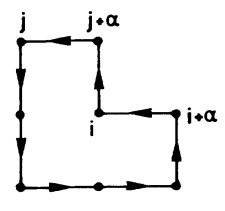

(c)
FIG. 16. Graphs contributing to $f^{X}$. 
We will now perform the explicit evaluations of $\sigma^{X}(\omega)$ in the Néel and random backgrounds.

\section{Néel background}

The Green function $G^{N}$ of a hole moving in a Néel spin configuration was shown to be diagonal in $d=\infty$. This property remains in the case of $G^{N}$ derived within our approximation and the retraceable-path approximation in $d<\infty$. Hence the only contribution to $f^{N}$ (see Fig. 15) comes from the graph shown in Fig. 17, where the bold lines represent the diagonal Green function $G^{N}$, which starts and ends at the same lattice point. This graph is obtained from that shown in Fig. 15(a) by identifying site $\mathrm{j}$ with $\mathrm{i}-\boldsymbol{\alpha}$ and $\mathrm{i}$ with $\mathrm{j}+\boldsymbol{\alpha}$, respectively. The hole starts and ends on site $\mathbf{i}$. The spin on $\mathbf{j}$ may be up or down and is automatically restored by the type of path in Fig. 17. The algebraic expression corresponding to the graph in Fig. 17 reads

$$
f^{N}\left(z_{1}, z_{2}\right)=2 e^{2} a^{2} t^{2} L G^{N}\left(z_{1}\right) G^{N}\left(z_{2}\right),
$$

where (53) and (54) have been used to obtain the correct prefactors. This result has already been derived by Brinkman and Rice. ${ }^{9}$ Here we have shown that for a Néel spin background it is exact in high dimensions. From (49) and (55) one finds, for the function $F^{N}$,

$$
F^{N}\left(\omega_{1}, \omega_{2}\right)=-8 \pi^{2} e^{2} a^{2} t^{2} L D^{N}\left(\omega_{1}\right) D^{N}\left(\omega_{2}\right),
$$

which can be inserted into (48) or (51) to calculate the conductivity $\sigma^{N}(\omega)$. In the zero-temperature limit $T \rightarrow 0$, one obtains the simple result

$$
\operatorname{Re} \sigma^{N}(\omega)=\frac{2 \pi e^{2} a^{2} t^{2}}{V \omega} D^{N}\left(\omega-\omega_{0}\right),
$$

where $-\omega_{0}$ is the lower band edge of $D^{N}$ (e.g., $\omega_{0}=2 t^{*}$ in $d=\infty)$. Inserting $D^{N}$ [Eq. (21)] into (56), we obtain the exact expression for the conductivity of a hole moving in a Néel spin background in $d=\infty$. Since the conductivity is a correlation function between two sites (i.e., for a given direction $\alpha$ ), it vanishes as $1 / d$ for $d \rightarrow \infty$ as a result of $t^{2}=t^{* 2} / 2 d$. However, $\sigma / t^{2} \propto \sigma d$ remains finite in $d=\infty$ and can be compared with results in $d<\infty$. The result for $\operatorname{Re} \sigma^{N}(\omega)$ in $d=\infty$ is plotted in Fig. 18 for various temperatures. The dc conductivity $\operatorname{Re} \sigma^{N}(0)$ diverges only at zero temperature. Of course, our exact result for $\operatorname{Re} \sigma^{N}(\omega)$ obeys the $f$-sum rule ${ }^{31}$

$$
\int_{-\infty}^{\infty} \operatorname{Re} \sigma(\omega) d \omega=-\frac{\pi e^{2} a^{2}}{V}\left\langle H_{\alpha}\right\rangle
$$

where $\left\langle H_{\alpha}\right\rangle=\langle H\rangle / d$ is the average kinetic energy in one particular direction $\alpha$.

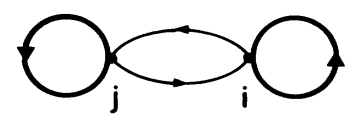

FIG. 17. Graph contributing to $f^{N}$; the bold lines represent the Green function $G^{N}$.

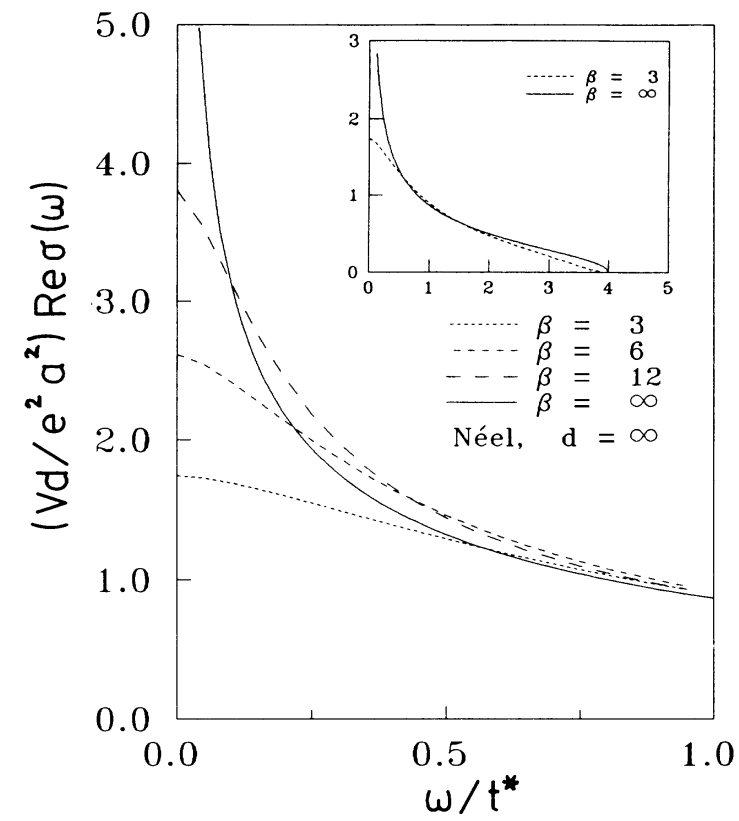

FIG. 18. Exact dynamical conductivity for a hole in a Néel background, $\operatorname{Re} \sigma^{N}(\omega)$, for various temperatures $\left(\beta=1 / k_{B} T\right)$ in $d=\infty$. All energies are scaled by $t^{*}$. In the inset the behavior for larger $\omega$ is shown. The conductivity vanishes at $\omega=4 t^{*}$, corresponding to the bandwidth.

\section{Random background}

In $d=\infty, f_{2}^{X}$ is zero for arbitrary backgrounds (see Sec. V B). Summing the noninterfering segments 2 and 4 of the graph in Fig. 15(a), one finds the exact result for $f_{1}^{R}$ in $d=\infty$, namely,

$f_{1}^{R}\left(z_{1}, z_{2}\right)=2 e^{2} a^{2} t^{2} \sum_{\sigma} \sum_{\mathrm{i}, \mathrm{j}} \mathrm{p}_{\sigma}^{-1} \mathrm{G}_{\sigma \mathrm{ji}}^{\mathrm{R}}\left(\mathrm{z}_{1}\right) \mathrm{G}_{\sigma \mathrm{ij}}^{\mathrm{R}}\left(\mathrm{z}_{2}\right)$,

where the prefactor has been obtained by (53) and the site indices have been shifted because of translational invariance. The requirement of spins to be equal for a loop to contribute in $d=\infty$ is satisfied owing to the additional factor $p_{\sigma}^{-1}$ in (59). Inserting (59) in (49) and using the Fourier representation of $G_{\sigma \mathrm{ij}}^{R}(z)$, one obtains

$$
F^{R}\left(\omega_{1}, \omega_{2}\right)=-8 \pi^{2} e^{2} a^{2} t^{2} \sum_{\sigma} \sum_{\mathbf{k}} p_{\sigma}^{-1} \rho_{\sigma}^{R}\left(\mathbf{k}, \omega_{1}\right) \rho_{\sigma}^{R}\left(\mathbf{k}, \omega_{2}\right)
$$

Inserting $F^{R}$ into (51) and integrating (numerically) over $\omega_{1}$, one can now calculate the exact conductivity for a hole moving in a random spin background in $d=\infty$.

We note that within standard perturbation theory and for a grand canonical ensemble of interacting lattice fermions in $d=\infty$, the conductivity is determined exactly by the simple bubble diagram; i.e., vertex corrections vanish. ${ }^{32}$ In our case (one hole in a restricted Hilbert space), the applicability of this standard formalism is not a priori guaranteed, but eventually we obtain the same result.

In the ferromagnetic limit $\left(p_{\sigma} \rightarrow 1, p_{-\sigma} \rightarrow 0\right)$, the spectral function $\rho_{\sigma}^{F}$ is a $\delta$ function, $\rho_{\sigma}^{F}(\mathbf{k}, \omega)=\delta\left(\omega+\varepsilon_{\mathbf{k}}\right)$, while $\rho_{-\sigma}^{F}$ is zero, and (60) becomes 


$$
F^{F}\left(\omega_{1}, \omega_{2}\right)=-8 \pi^{2} e^{2} a^{2} t^{2} L D^{0}\left(\omega_{1}\right) \delta\left(\omega_{1}-\omega_{2}\right) .
$$

The real part of the conductivity therefore becomes

$$
\operatorname{Re} \sigma^{F}(\omega)=2 \pi e^{2} a^{2} t^{2} V^{-1} \beta \delta(\omega) .
$$

This is, of course, precisely the result for a free particle on an empty lattice in $d=\infty$.

Returning to the unpolarized random background, the dc conductivity $\operatorname{Re} \sigma^{R}(0)$ is infinite for all temperatures, but the corresponding peak contains considerable spectral weight only at low temperatures. At $T=0$ only a $\delta$ peak at $\omega=0$ remains, i.e.,

$\lim _{T \rightarrow 0} \operatorname{Re} \sigma^{R}(\omega)=\frac{\pi e^{2} a^{2} t^{2}}{2 V} \beta \delta(\omega) 2 e^{2} t^{2} \beta \delta(\omega)=\frac{1}{4} \operatorname{Re} \sigma^{F}(\omega)$.

The prefactor has been calculated from the $f$-sum rule, which must necessarily be satisfied in this exact calculation (and has indeed been checked numerically at high temperatures, where the peak at $\omega=0$ carried only negligible weight). The origin of the low-T behavior of $\sigma^{R}$ is quite clear. At low temperatures only the low-energy states are relevant. In Sec. III we found that single-hole states in the random background with low energy $(\omega \rightarrow-\infty)$ behave like free-particle states with a dispersion $-\varepsilon_{\mathrm{k}} / 2$. It is therefore not surprising that $\sigma^{R}(\omega)$ is given by a free-particle conductivity with $t$ substituted by $t / 2$ for $T \rightarrow 0$.

E. Sum-rule-conserving approximation for finite dimensions

\section{Néel background}

As in the case of the density of states (see Sec. III E), the conductivity $\sigma(\omega)$ in $d<\infty$ may be calculated by two different approximations, both of which become exact in $d=\infty$. Namely, the density of states determining the partition function $Z^{X}$ as well as $F^{X}$ in (56) can either be approximated by that obtained within the retraceablepath approximation or by the half-ellipse [Eq. (21)]. In the latter case the $f$-sum rule (58) is fulfilled exactly for all $d$ (again, the $d$ dependence only enters through $t^{*} / \sqrt{2 d}$ ). By contrast, for $d<\infty$ the retraceable-path approximation never fulfills this sum rule as was noticed for $d=2$ in Ref. 11. This can be explicitly verified in the limit $T \rightarrow 0$. Using (57), the right-hand side of (58) yields

$$
\int_{-2 \omega_{c}^{*}}^{2 \omega_{c}^{*}} d \omega \operatorname{Re} \sigma^{\mathrm{rpa}}(\omega)=\frac{\pi}{d-1} e^{2} \frac{a^{2}}{V} \omega_{c}^{*},
$$

where $\omega_{c}^{*}=\sqrt{2(2 d-1) / d} t^{*}$ is half the bandwidth of the density of states $D^{\text {rpa }}$. On the other hand, the right-hand side of (58) is given by $-\pi e^{2} a^{2} \omega_{c}^{*} / V d$. Hence the ratio of both sides is $(d-1) / d$, showing that the $f$-sum rule is only exactly fulfilled in the limit $d=\infty$. The conductivity $\sigma(\omega)$ for a hole in $d=3$ as obtained by the retraceablepath approximation is shown in Fig. 19. This result should be compared with the exact result in $d=\infty$ shown in Fig. 18, which, as explained in Sec. III E, can also be employed as a sum-rule-conserving approximation for $\sigma^{N}$ in $d<\infty$. Although the curves are qualitatively very similar, the scales of $\sigma(\omega)$ are seen to differ by about a factor of 2 for $T=0$ and $\omega / t^{*}<1$. More precisely, in

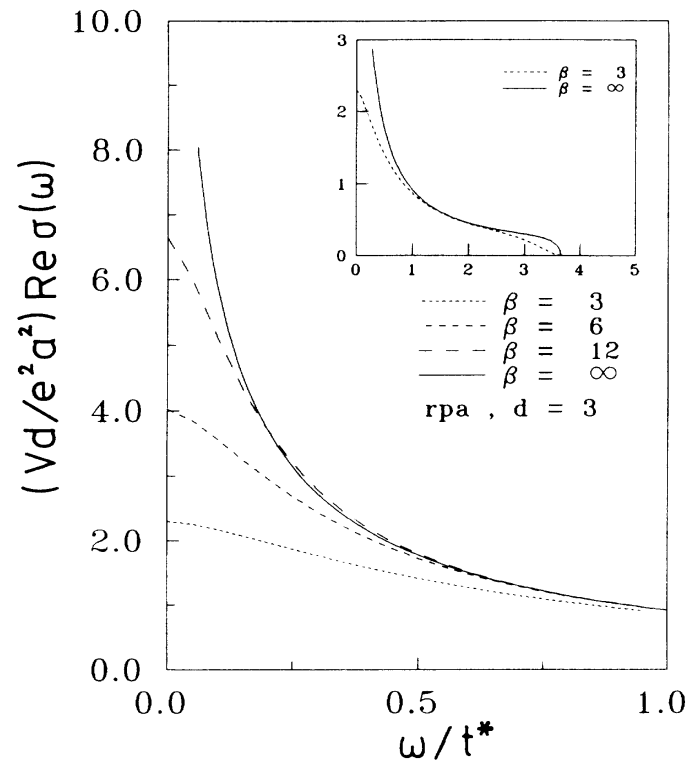

FIG. 19. Dynamical conductivity $\operatorname{Re} \sigma(\omega)$ for a hole in $d=3$ for various temperatures $\left(\beta=1 / k_{B} T\right)$, as obtained by the retraceable-path approximation (rpa).

this limit the retraceable-path approximation yields

$$
\begin{aligned}
\operatorname{Re} \sigma^{\mathrm{rpa}}(\omega) & =\frac{9}{8}\left[\frac{40}{3}\right]^{1 / 4} \frac{e^{2}}{d} \frac{a^{2}}{V}\left(\frac{t^{*}}{\omega}\right)^{1 / 2} \\
& \simeq 2.15 \frac{e^{2}}{d} \frac{a^{2}}{V}\left[\frac{t^{*}}{\omega}\right]^{1 / 2},
\end{aligned}
$$

while our approximation for the Néel state leads to

$$
\operatorname{Re} \sigma^{N}(\omega)=\frac{e^{2}}{d} \frac{a^{2}}{V}\left[\frac{t^{*}}{\omega}\right]^{1 / 2} .
$$

The different prefactors originate from the different shape and position of the band edges of the density of states in the two cases (see Fig. 10). It is this dependence which is of crucial quantitative significance for $\sigma(\omega)$ (particularly at low temperatures), since the latter is determined by an integral over the product of two shifted densities of states whose main contribution comes from the region close to the band edges. The fact that the density of states obtained by the retraceable-path approximation is correct up to $O\left(1 / d^{4}\right)$ (cf. the discussion in Sec. III E) clearly does not imply that the corresponding $\sigma(\omega)$, or at least the $f$-sum rule, is given by a similar degree of accuracy. Therefore, our approximation for $\sigma^{N}(\omega)$, which fulfills the $f$-sum rule exactly, can be expected to be more accurate in this case.

\section{Random background}

In $d<\infty$ the term $f_{2}^{X}$ [Eq. (54)] contributes, too. Its presence is necessary to treat the ferromagnetic limit correctly in $d<\infty$. However, we still neglect interferences between the segments 2 and 4 of the hole paths illustrated in Fig. 15; for $d<\infty$ this is an approximation. Hence, summing the contributions to the segments 2 and 4 in Fig. 15(b) separately and using (54), one obtains 


$$
f_{2}^{R}\left(z_{1}, z_{2}\right)=-\frac{e^{2} a^{2}}{d} t^{* 2} \sum_{\sigma} \sum_{\mathrm{ij}} p_{\sigma}^{-1} G_{\sigma, \mathrm{j}+\alpha, \mathrm{i}}^{R}\left(z_{1}\right) G_{\sigma, \mathrm{i}+\alpha, \mathrm{j}}^{R}\left(z_{2}\right) .
$$

Adding this to $f_{1}^{R}$ [Eq. (59)] and Fourier representing $G_{\sigma}^{R}$, one finds

$$
f^{R}\left(z_{1}, z_{2}\right)=\frac{e^{2} a^{2}}{d} t^{* 2} \sum_{\sigma} p_{\sigma}^{-1} \sum_{\mathbf{k}} 2 \sin ^{2} k_{\alpha} G_{\sigma}^{R}\left(\mathbf{k}, z_{1}\right) G_{\sigma}^{R}\left(\mathbf{k}, z_{2}\right)
$$

where $k_{\alpha}$ is the component of $\mathbf{k}$ in direction $\alpha$. In $d \rightarrow \infty$, (67) reduces to (59) since $G_{\sigma}^{R}$ depends on $\mathbf{k}$ only via $\varepsilon_{\mathbf{k}}$; i.e., $\sin ^{2} k_{\alpha}$ can be substituted by its average value $\frac{1}{2}$ because $\mathbf{k}$ and $\varepsilon_{\mathrm{k}}$ are uncorrelated in $d \rightarrow \infty .{ }^{21}$ Inserting $f^{R}$ [Eq. (67)] in (49), we obtain

$$
F^{R}\left(\omega_{1}, \omega_{2}\right)=-\frac{4 \pi^{2} e^{2} a^{2}}{d} t^{* 2} \sum_{\sigma} p_{\sigma}^{-1} \sum_{\mathbf{k}} 2 \sin ^{2} k_{\alpha} \rho_{\sigma}^{R}\left(\mathbf{k}, \omega_{1}\right) \rho_{\sigma}^{R}\left(\mathbf{k}, \omega_{2}\right)
$$

which, via (48) or (51), yields our approximate result for the conductivity $\sigma^{R}(\omega)$ in $d<\infty$.

In the ferromagnetic limit $p_{\sigma} \rightarrow 1, p_{-\sigma} \rightarrow 0$, the conductivity obtained within this approximation is

$$
\sigma^{F}(\omega)=-\frac{\pi e^{2}}{d} \frac{a^{2}}{V}\langle H\rangle_{F} \delta(\omega),
$$

where $\langle H\rangle_{F}$ is the average kinetic energy of a hole in the ferromagnetic background. This result is exact for all $d$.

The complex conductivity $\sigma^{R}(\omega)$ calculated from (48) and (68) is automatically analytic for $\operatorname{Im} \omega \neq 0$, and $\operatorname{Re} \sigma^{R}(\omega)$, given by (51) and (68), is always positive or zero (for real $\omega$ ), in agreement with the corresponding exact properties of the dynamical conductivity.

Results for the conductivity in an unpolarized $\left(p_{\uparrow}=p_{\downarrow}=\frac{1}{2}\right)$ random spin background in $d=3$, as calculated from (68), are shown in Fig. 20; for $\rho^{R}$ the approximate result from Sec. III E has been inserted. For high temperatures, $\sigma^{R}(\omega)$ is a broad function which is finite at

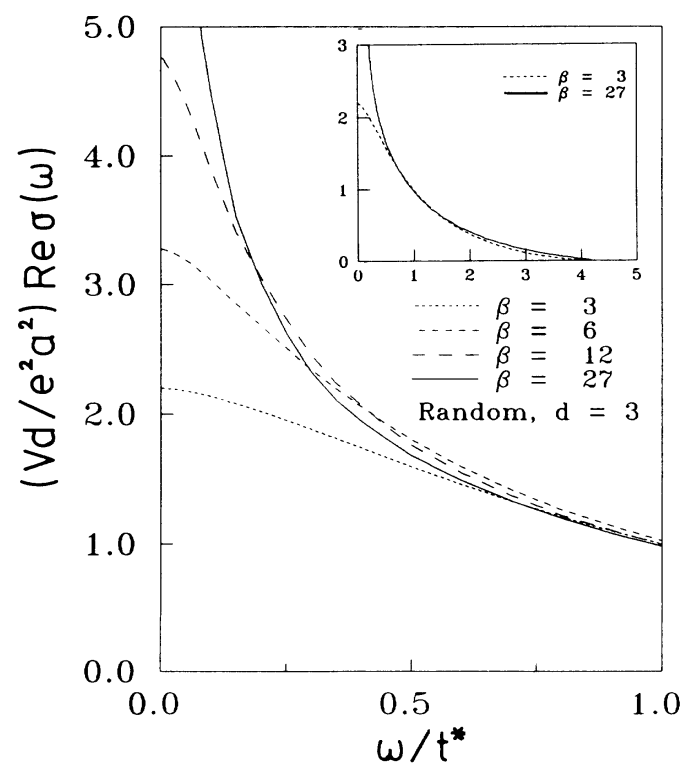

FIG. 20. Dynamical conductivity for a hole in an unpolarized random background, $\operatorname{Re} \sigma^{R}(\omega)$, for various temperatures $\left(\beta=1 / k_{B} T\right)$ in $d=3$. $\omega=0$. At lower temperatures, more and more spectral weight moves into the neighborhood of $\omega=0$, and at $T \rightarrow 0, \sigma^{R}(0)$ diverges.

The exact conductivity $\sigma^{R}(\omega)$ at $T=0$ should actually be a $\delta$ function in $d=3$ (as in $d=\infty$ ), since at low $T$ the Boltzmann factor in the Kubo formula singles out spin configurations with large ferromagnetic clusters around the hole. These clusters are not well described by our approximation, and consequently the expected $\delta$ peak is not reproduced in $d<\infty$. The corresponding dc electrical resistivity $\rho(T)$ of a hole in $d=3$ is shown in Fig. 21 for different backgrounds. The result for the retraceablepath approximation has already been obtained earlier by Oguri and Maekawa. ${ }^{12}$ The resistivity is seen to be almost linear for almost all $T$.

We conclude this section with a simple physical interpretation of our approximation for the conductivity. Consider a hole (or particle) moving in a medium which damps its motion while the medium itself is not affected by the motion; the damping, described by $\operatorname{Im} \Sigma^{R}\left(\omega-i 0^{+}\right)$, may have an arbitrary energy dependence. In this situation Eq. (68), which relates the con-

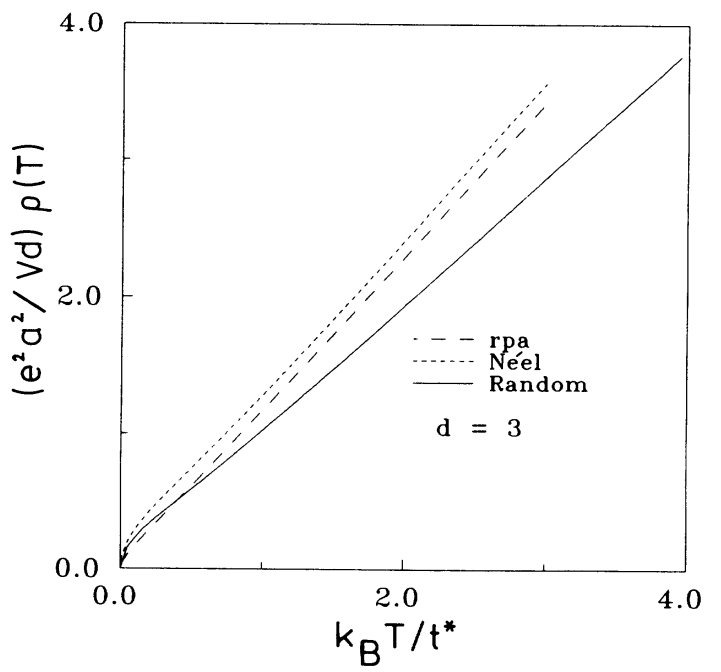

FIG. 21. Electrical resistivity $\rho(T)$ for a hole in $d=3$ for various background configurations (Néel, random) in comparison with the retraceable-path approximation (rpa). 
ductivity to the spectral function $\rho^{R}$, is exact, since the neglected self-interference of the hole path is irrelevant when the hole does not change the background. Having thus constructed a model for which (68) becomes exact (for any $\rho^{R}$ ), we can immediately conclude that the $f$-sum rule $[\mathrm{Eq} .(58)]$ is conserved by $(68)$.

\section{SUMMARY}

We have calculated the Green function, thermodynamics, and dynamical conductivity of a single hole moving in a spin background. The well-known retraceable-path approximation, where loops in the path of the hole are neglected, has been shown to be exact for a hole in a Néel background in high dimensions $d \rightarrow \infty$. However, for a general background this neglect leads to serious shortcomings in any dimension $d>1$; in particular, the hole dynamics does not depend on the spin configuration at all then. A theoretical approach, in which loops are included self-consistently to all orders, has been formulated. The resulting approximation scheme is sensitive to the spin configuration, becomes exact in high dimensions for any spin background, and treats the case of a ferromagnetic background exactly in any dimension; the spectral sum rule for the Green function and the $f$-sum rule for the conductivity are conserved. In view of the starting point of our approximation, i.e., the limit $d \rightarrow \infty$, our results are expected to apply to system in dimensions $d \geq 3$. In particular, quantum spin fluctuations, which are not treated here, become increasingly important as one goes to lower dimensions and are of fundamental relevance in $d=1,2$.

Three types of spin backgrounds have been discussed explicitly: ferromagnetic, Néel, and random, where in the latter case all configurations are thermodynamically averaged. Within our calculation, performed explicitly in $d=3$ and $\infty$, the density of states of a hole in a Néel or random background is found to become narrower with respect to the ferromagnetic case, the narrowing being stronger for the Néel case. The spectral function is $\mathbf{k}$ dependent except for the Néel background, but has quasiparticle peaks only in the ferromagnetic case. The spinconfiguration dependence is particularly pronounced for the dynamical conductivity $\operatorname{Re} \sigma(\omega)$. For a hole in a ferromagnetic background, we recover the exact result; i.e., the conductivity is a $\delta$ function at all temperatures $T$ and for all $d$. In the Néel case the conductivity is found to be a smooth function which is finite in $\omega=0$ for $T>0$, while at $T=0$ there is a $\omega^{-1 / 2}$ singularity for small $\omega$ (both in $d=3$ and $\infty$ ). The conductivity for a hole in the infinitedimensional random background is always infinite at $\omega=0$, but the singularity is very weak at high temperatures; for $T>0$ it is nonzero also at $\omega \neq 0$, while at $T=0$ all spectral weight is absorbed in a $\delta$ peak at $\omega=0$. By contrast, in $d=3$ our result for the dc conductivity is infinite only at $T=0$ and the ac conductivity is nonzero for all $T \geq 0$. Our approach, as any previous one, does not give a good description of the contribution of large ferromagnetic clusters in the random background at low temperatures in $d<\infty$. Correspondingly, the expected Lifshitz tails in the density of states and the $\delta$ peak in the zero-temperature conductivity are not obtained by our approximation for $d<\infty$. Unfortunately, there are no numerical studies of the hole dynamics in $d=3$, where our theory, being exact in $d=\infty$, should be particularly accurate.

There are several applications and possible extensions of our results. For the polarized random spin background, they can be directly applied to the calculation of the magnetoresistence and Hall effect, where a proper summation of loops is crucial. As a straightforward generalization, the above theory, which has been derived for three special types of spin backgrounds, can be easily extended to any mean-field ensemble of spin configurations in a Heisenberg model. The spin excitation energy, which has not been considered here, can also be implemented in our approach. A practical approximate treatment of spin fluctuations may be achieved by summing the same class of hole paths as above, but allowing for arbitrary, background-restoring spin flips along the path.

\section{ACKNOWLEDGMENTS}

One of us (D.V.) wishes to thank P. Horsch for correspondence on recent numerical studies of the hole problem in $d=1,2$ and E. Müller-Hartmann for a discussion on the stability of the Nagaoka state. This work was supported in part by the Sonderforschungsbereich 341 of the Deutsche Forschungsgemeinschaft.

\section{APPENDIX: ANALYTIC PROPERTIES OF $G^{R}$ AND SPECTRAL SUM RULE}

In this appendix we show that our result for $G^{R}(\mathbf{k}, z)$ has the following properties.

(i) $G^{R}(\mathbf{k}, z)$ is analytic for $\operatorname{Im} z>0$ and $\operatorname{Im} z<0$.

(ii) $\rho^{R}(\mathbf{k}, z)$ is never negative.

(iii) The spectral sum rule (41) is satisfied.

In $d=\infty$ the Green function has been determined exactly; therefore, it satisfies the exact properties (i)-(iii) $a$ priori.

In $d<\infty$, where $D^{0}$ has a finite bandwidth, the power series (12) converges if $|z|$ is larger than some value $z_{0}$ (this is easily shown by deriving upper bounds for the number of $n$-step hole paths; cf. Ref. 10). Hence $G^{R}(z)$, as obtained from (32), is analytic for $|z|>z_{0}$, since in this case it is given by the sum of a convergent power series in $1 / z$. For $|z|<z_{0}$, Eq. (32) determines an analytic continuation of $G^{R}(z)$. This continuation has singularities only on the real axis. This is because (32) has no real solution $G^{R}$ for complex $z$ [real $G^{R}$ implies real $z$ by (32), since $G_{-1}^{0}(\xi)$ is real for real $\left.\xi\right]$, while near a singularity (pole, essential singularity, branch cut) real values must exist. Hence $G^{R}(z)$ is analytic for $\operatorname{Im} z \neq 0$.

Since $G^{R}(z)$ is complex for complex $z, \operatorname{Im} G^{R}(z)$ does not change sign in the upper and lower complex $z$ planes, respectively. For large $|z|$, Eq. (32) has the asymptotic solution

$$
G^{R}(z)^{|z|} \underset{\sim \infty}{\sim} \frac{1}{z},
$$

which identifies the sign of $\operatorname{Im} G^{R}(z)$ as 


$$
\begin{aligned}
& \operatorname{Im} G^{R}(z)<0 \text { for } \operatorname{Im} z<0, \\
& \operatorname{Im} G^{R}(z)>0 \text { for } \operatorname{Im} z>0 .
\end{aligned}
$$

Consequently, by (9) and (36),

$$
\begin{aligned}
& \operatorname{Im} G^{R}(\mathbf{k}, z)<0 \text { for } \operatorname{Im} z<0, \\
& \operatorname{Im} G^{R}(\mathbf{k}, z)>0 \text { for } \operatorname{Im} z>0 .
\end{aligned}
$$

Hence, by the same arguments as for $G^{R}(z)$ above, $G^{R}(\mathbf{k}, z)$ is analytic for $\operatorname{Im} z>0$ and $\operatorname{Im} z<0$; this proves (i). Furthermore, (A3) yields (ii), namely, $\rho^{R}(\mathbf{k}, \omega)=\pi^{-1} G^{R}\left(\mathbf{k}, \omega-i 0^{+}\right) \geq 0$.

Since $G^{R}(\mathbf{k}, z)$ is analytic for $\operatorname{Im} z \neq 0$, it can be expressed by Cauchy's integral formula as

$$
G^{R}(\mathbf{k}, z)=\frac{1}{2 \pi i} \int_{\Gamma} \frac{G^{R}(\mathbf{k}, \zeta)}{z-\zeta} d \zeta,
$$

where $\Gamma$ is a contour enclosing the singularities of
$G^{R}(\mathbf{k}, \zeta)$ on the real axis and $z$ is not enclosed by $\Gamma$. Equations (36), (32), and (9) imply that

$$
G^{R}(\mathbf{k}, \bar{z})=\bar{G}^{R}(\mathbf{k}, z),
$$

where the bar indicates complex conjugation. Choosing the contour $\Gamma$ arbitrarily close to the real axis, one obtains from (A4) and (A5), for $\operatorname{Im} z \neq 0$,

$$
G^{R}(\mathbf{k}, z)=\frac{1}{\pi} \int_{-\infty}^{\infty} d \omega \frac{\operatorname{Im} G^{R}\left(\mathbf{k}, \omega-i 0^{+}\right)}{z-\omega} .
$$

For large $|z|$, (A6) yields

$$
\int_{-\infty}^{\infty} d \omega \rho^{R}(\mathbf{k}, \omega)=\lim _{z \rightarrow \infty} z G^{R}(\mathbf{k}, z) .
$$

Integrating (A7) over $\mathbf{k}$ and using (A1) for the right-hand side, one obtains the spectral sum rule (41). The positivity of $\rho^{R}$ and the spectral sum rule have also been checked numerically for several cases.
${ }^{1}$ J. G. Bednorz and K. A. Müller, Z. Phys. B 64, 189 (1986).

${ }^{2}$ P. W. Anderson, Science 235, 1196 (1987).

${ }^{3}$ M. C. Gutzwiller, Phys. Rev. Lett. 10, 159 (1963); J. Hubbard, Proc. R. Soc. London, Ser. A 276, 238 (1963); J. Kanamori, Prog. Theor. Phys. 30, 275 (1963).

${ }^{4}$ A. B. Harris and R. V. Lange, Phys. Rev. 157, 295 (1967).

${ }^{5}$ See, for example, F. C. Zhang and T. M. Rice, Phys. Rev. B 37, 3759 (1988).

${ }^{6}$ B. J. Shraiman and E. D. Siggia, Phys. Rev. Lett. 60, 740 (1988).

${ }^{7}$ V. J. Emery, S. A. Kivelson, and H. Q. Lin, Phys. Rev. Lett. 64, 475 (1990).

${ }^{8}$ M. Ogata, M. V. Luchini, S. Sorella, and F. F. Assaad, Phys. Rev. Lett. 66, 2388 (1991).

${ }^{9}$ W. F. Brinkman and T. M. Rice, Phys. Rev. B 2, 1324 (1970).

${ }^{10}$ Y. Nagaoka, Phys. Rev. 147, 392 (1966).

${ }^{11}$ T. M. Rice and F. C. Zhang, Phys. Rev. B 39, 815 (1989).

${ }^{12}$ A. Oguri and S. Maekawa, Phys. Rev. B 41, 6977 (1990).

${ }^{13}$ S. Schmitt-Rink, C. M. Varma, and A. E. Ruckenstein, Phys. Rev. Lett. 60, 2793 (1988).

${ }^{14}$ C. L. Kane, P. A. Lee, and N. Read, Phys. Rev. B 39, 6880 (1989).

${ }^{15}$ C. Gros and M. Johnson, Phys. Rev. B 40, 9423 (1989).

${ }^{16}$ S. Trugman, Phys. Rev. B 41, 892 (1990).

${ }^{17}$ K. J. v. Szczepanski, P. Horsch, W. Stephan, and M. Ziegler, Phys. Rev. B 41, 2017 (1990).

${ }^{18}$ E. Dagotto, R. Joynt, A. Moreo, S. Bacci, and E. Gagliano, Phys. Rev. B 41, 9049 (1990).

${ }^{19}$ J. Inoue and S. Maekawa, J. Phys. Soc. Jpn. 59, 2110 (1990).

${ }^{20}$ W. F. Brinkman and T. M. Rice, Phys. Rev. B 4, 1566 (1971).

${ }^{21}$ W. Metzner and D. Vollhardt, Phys. Rev. Lett. 62, 324 (1989).
${ }^{22}$ The stability of the ferromagnetic state in the thermodynamic limit and $\delta \rightarrow 0$ is a subtle question, even in $d \geq 3$, to which a final answer has not yet been given; see B. S. Shastry, H. R. Krishnamurty, and P. W. Anderson, Phys. Rev. B 41, 2375 (1990); T. Kopp, A. E. Ruckenstein, and S. Schmitt-Rink, ibid. 42, 6850 (1990); also W. von der Linden and D. M. Edwards, J. Phys. Condens. Matter 3, 4917 (1991), for $d=2$.

${ }^{23}$ The stability of the Nagaoka state has been discussed most recently for various lattice structures by $\mathrm{E}$. Müller-Hartmann (unpublished).

${ }^{24}$ V. Janiš and D. Vollhardt, Int. J. Mod. Phys. B (to be published).

${ }^{25}$ The Néel state is the exact ground state of the antiferromagnetic Heisenberg model in $d=\infty$ dimensions; see, for example, T. Kennedy, E. H. Lieb, and B. S. Shastry, Phys. Rev. Lett. 61, 2582 (1988).

${ }^{26}$ For reviews, see D. Vollhardt, Physica B 169, 277 (1991); E. Müller-Hartmann, Int. J. Mod. Phys. B 3, 2169 (1989).

${ }^{27}$ W. Metzner, Phys. Rev. B 43, 8549 (1991).

${ }^{28}$ For a discussion of analytic properties of Green functions, see, for example, J. W. Negele and H. Orland, Quantum Many Particle Systems (Addison-Wesley, New York, 1988).

${ }^{29}$ The quantity $S^{R}$ has also been introduced in Ref. 9 where it was referred to as self-energy $\Sigma$; we use a different symbol to avoid confusion with the self-energy we introduced in (9), which is different from $S^{R}$.

${ }^{30}$ I. M. Lifshitz, Adv. Phys. 13, 483 (1964).

${ }^{31}$ D. Baeriswyl, C. Gros, and T. M. Rice, Phys. Rev. B 35, 8391 (1987).

${ }^{32}$ A. Khurana, Phys. Rev. Lett, 64, C1990 (1990). 